\title{
Conic Martingales from Stochastic Integrals
}

\author{
Frédéric VRINS* and Monique JEANBLANC ${ }^{\dagger}$
}

* Université catholique de Louvain

Louvain School of Management

Chaussée de Binche 151, 7000 Mons, Belgium

$\&$

Center for Operational Research and Econometrics (CORE)

Voie du Roman Pays 20, 1348 Louvain-la-Neuve, Belgium

† Université d'Évry Val d'Essonne

Laboratoire de Mathématiques et Modélisation d'Évry (LaMME)

UMR CNRS 8071, Évry, France

October 21, 2018

\begin{abstract}
In this paper we introduce the concept of conic martingales. This class refers to stochastic processes having the martingale property, but that evolve within given (possibly time-dependent) boundaries. We first review some results about the martingale property of solution to driftless stochastic differential equations. We then provide a simple way to construct and handle such processes. Specific attention is paid to martingales in $[0,1]$. One of these martingales proves to be analytically tractable. It is shown that up to shifting and rescaling constants, it is the only martingale (with the trivial constant, Brownian motion and Geometric Brownian motion) having a separable coefficient
\end{abstract}

${ }^{*}$ Contact author. E-mail: frederic.vrins@uclouvain.be ${ }^{\dagger}$ The research of Monique Jeanblanc is supported by the Chaire Marchés en Mutation (Fédération Bancaire Française). 
$\sigma(t, y)=g(t) h(y)$ and that can be obtained via a time-homogeneous mapping of Gaussian diffusions.

The approach is exemplified to the modeling of stochastic conditional survival probabilities in the univariate (both conditional and unconditional to survival) and bivariate cases.

Keywords : Bounded martingale, stochastic differential equation, diffusion process, stochastic survival probability

\section{Introduction and motivation}

Mathematical finance extensively relies on martingales, mainly due to the Fundamental Theorem of Asset Pricing. For instance, they are used to represent the dynamics of (non-dividend paying) asset prices, denominated in units of numéraire under an adequate associated measure. As a consequence, under a true martingale condition, asset price processes are governed by conditional risk-neutral expected values of future (discounted) cashflows. Martingales are also central in measure change techniques (via Radon-Nikodym derivative processes).

Depending on the situation, the martingale processes may be subjected to some constraints. Discounted stock prices and Radon-Nikodym derivative processes are positive. Therefore, exponential martingales, which meet the non-negativity constraint are very popular tools.

Financial processes can be subjected to other constraints, like being bounded below and above. This is for instance the case of discounted zero-coupon bond prices in the case where interest rates (short rate $r_{s}$ ) cannot be negative:

$$
P_{t}(T)=\mathbb{E}\left[\exp -\int_{t}^{T} r_{s} d s \mid \mathcal{F}_{t}\right],
$$

which belongs to $[0,1]$ almost surely, and thus so is the martingale $P_{t}(T) \mathrm{e}^{-\int_{0}^{t} r_{s} d s}$. Similarly, conditional survival probabilities $S_{t}(T)$ (probability that a default event $\tau$ occurs after a given time $T$ as seen from time $t$ ), defined as the conditional expected value of survival indicators

$$
S_{t}(T)=\mathbb{E}\left[\mathbb{I}_{\{\tau>T\}} \mid \mathcal{F}_{t}\right]
$$

are $\left(\mathcal{F}_{t}\right)_{t \geqslant 0}$-martingales valued in $[0,1]$. Note that here, we have to deal with a family of martingales depending on the parameter $T$ and that $S_{t}(T)$ has to be, for any $t$, decreasing with respect to $T$. Note that $\mathbb{E}\left[S_{t}(T)\right]=\mathbb{Q}\{\tau>T\}=S_{0}(T)$.

Surprisingly however, bounded martingales received little attention. In the case of survival probabilities modeling for instance, practitioners often disregard inconsistencies, working with Gaussian processes (which are not constrained to evolve in the unit interval) instead (see e.g. Cesari et al. (2009)). This 
also applies to many standard approaches, where shifted Ornstein-Uhlenbeck (Hull-White) or shifted square-root diffusion (SSRD, also known as $\mathrm{CIR}^{++}$) are used as intensity processes, and may lead to probabilities exceeding $1 .{ }^{1}$

In spite of these drawbacks, these methods remain popular. We believe this results from the lack of adequate and tractable alternatives. Our purposes here is precisely to propose a contribution to fill this gap. We introduce the concept of conic martingales (a naming that we justify in the sequel), which corresponds to the intuitive idea of "martingales evolving between bounds". We shall see how such processes can be handled and simulated in such a way that the paths stay within the bounds. We further study some properties like the implied distribution and the asymptotic behavior. Specific interest is dedicated to martingales obtained by mapping Gaussian processes through functions with image being a compact set.

The paper is an extension of Vrins (2014); Vrins and Jeanblanc (2015), and is organized as follows. We first sketch the model setup in Section 2 and recall some results related to existence, uniqueness and martingale property of driftless stochastic differential equations (SDE) in Section 3. The concepts of cone and conic martingales are then introduced in Section 4. We then discuss how those can be constructed in Section 5 and focus on one particular process (Section 6). Finally, we apply this process to the modeling of univariate (conditional and unconditional) and bivariate survival probability modeling in Section 7 before concluding.

\section{Setup}

We consider a filtered probability space $\left(\Omega, \mathcal{F}, \mathbb{F}=\left(\mathcal{F}_{t}\right)_{t \geqslant 0}, \mathbb{Q}\right)$ where $\mathbb{F}$ is a Brownian filtration hence, any martingale is continuous. In the sequel, all the processes are defined on $(\Omega, \mathbb{F})$. We study the martingale property of the solution $Y$ to a "driftless" SDE of the form

$$
d Y_{t}=\sigma\left(t, Y_{t}\right) d W_{t}, 0 \leqslant t \leqslant T
$$

where $W$ is a $\mathbb{Q}$-Brownian motion adapted to the filtration $\mathbb{F}$ and, for each $t$, the random variable $Y_{t}$ is restricted to be in some interval.

In the sequel, we shall omit the specification " $0 \leqslant t \leqslant T$ " in case of non-ambiguity, and the diffusion coefficient function $\sigma(t, y):[0, T] \times \mathbb{R} \rightarrow A \subseteq \mathbb{R}$ is always assumed to be continuous in $y$ and Borelmeasurable in $t$.

\footnotetext{
${ }^{1}$ The shift in the square-root process is required in order to fit CDS quotes, and may indeed affect the positivity of the resulting stochastic intensity.
} 
The process $Y$ is a local martingale, but may fail to be a martingale. Additional technical conditions are required to prevent the use of "too fancy" diffusion coefficient functions. However, $\sigma(.,$.$) does not need$ to be "that fancy" for the (global) martingale property to be lost. For instance, the solution to (2.1) is a martingale if $\sigma(t, x)=x$ (Geometric Brownian motion) but is a strict local martingale when $\sigma(t, x)=x^{2}$. The distinction between local and global martingale is crucial in financial applications in order to prevent arbitrage opportunities and bubbles Cox and Hobson (2005), Protter (2013). To determine whether the solution to the above SDE is indeed a martingale, the following square integrability condition can be useful (see e.g., Karatzas and Shreve (2005), Revuz and Yor (1999) and Section 4.9 of Shreve (2004))

Theorem 2.1. The stochastic process $\int_{0}^{\cdot} \sigma\left(s, Y_{s}\right) d W_{s}$ is a martingale on $[0, T]$ if

$$
\mathbb{E}\left[\int_{0}^{T} \sigma^{2}\left(s, Y_{s}\right) d s\right]<\infty
$$

Condition (2.2) may be difficult to check, since it requires to have some information about the solution to the SDE (which may even not exist). In this context, not a lot can be said at this stage about the martingale property of $Y$. However, some useful results can be found, based on the shape of the diffusion coefficient $\sigma(t, y)$ only, as reviewed in the next section.

\section{General results on martingale property of Itô stochastic in- tegrals}

In this section, the martingale property of $Y$ is discussed form the properties of the deterministic diffusion coefficient function $\sigma$. The main result in that respect is the following (Theorem 2.9 in Karatzas and Shreve (2005)):

Theorem 3.1. Let $\sigma(t, y)$ be Lipschitz in y for all $t \geqslant 0$. In addition, suppose $\sigma$ satisfies the sub-linearity condition

$$
|\sigma(t, y)| \leqslant C(1+|y|)
$$

for some constant $C<\infty$, then eq. (2.1) has a pathwise unique (and thus strong) solution $Y$ satisfying $\mathbb{E}\left[\int_{0}^{T} Y_{s}^{2} d s\right]<\infty$. Moreover, $Y$ is a martingale.

Condition (3.1) aims at preventing explosion, while the Lipschitz constraint typically guarantees existence and uniqueness. In particular, the solution to (2.1) does not explode.

This result ensures that the solution to $d Y_{t}=g(t) Y_{t} d W_{t}$ is a martingale for bounded functions $g$ on $[0, T]$. However, it is not enough to ensure that the solution to the square-root driftless SDE 
$d Y_{t}=\sigma \sqrt{Y_{t}} d W_{t}$ is also a martingale $(\sqrt{x}$ fails to be Lipschitz in any interval containing 0$)$. The existence is formally proven in Zvonkin (1974) by replacing the Lipschitz continuity by a Holder-1/2 one.

The class of admissible diffusion coefficients in this theorem can be significantly extended by replacing the global Lipschitz condition by a local one. This covers quite a large class of coefficients since every continuously differentiable function is locally Lipschitz (see Kloeden and Platen (1999)). Yet another extension relies on the Yamada-Watanabe condition only (see e.g. (Kloeden and Platen, 1999) Section 4.5 p.134-135 and (Karatzas and Shreve, 2005) (Jeanblanc et al., 2007, Section 5.5.5)).

It is worth noting that a sufficient and necessary condition exists in the time-homogeneous case $\sigma(t, y)=\sigma(y)$ when $\sigma(y)=0$ for all $y \leqslant 0$. The positive process $\int_{0}^{\cdot} \sigma\left(Y_{s}\right) d W_{s}$ is a martingale if and only if $x / \sigma^{2}(x)$ is not integrable near infinity (see e.g. Carr et al. (2007), Delbaen and Shirakawa (2002)). These generalizations are however not necessary in the context of this paper.

\section{Cones of stochastic processes and conic martingales}

The above framework depicts the general context associated to the martingale property of solutions to driftless SDEs. We would like now to focus on bounded processes, and introduce the concept of cone.

Definition 4.1 (Bounded process). A stochastic process $Y$ is bounded on $[0, T]$ if there exists a constant $M$ such that $\sup _{t \in[0, T]}\left|Y_{t}\right|<M$. It is locally bounded on $\mathbb{R}$ if for all $t>0$ there exists a constant $M(t)$ such that $\sup _{s \leqslant t}\left|Y_{s}\right|<M(t)$.

We recall that the range $R(X)$ of a random variable $X$ is the support (which is a closed set, see (Dellacherie and P.-A., 1975, Ch. 3-50)) of its distribution. By extension, we define the range envelope of a stochastic process.

Definition 4.2 (Range envelope). The range envelope $\mathcal{S}:=\left(R\left(Y_{t}\right)\right)_{t>0}$ of a stochastic process $Y$ is the time-indexed sequence of the ranges $R\left(Y_{t}\right)$ of $\left(Y_{t}\right)_{t>0}$.

Observe that by continuity, the range envelope of a continuous process is a sequence of connected sets.

Definition 4.3 (Cone). If for each $t>0$ there exists $M(t)>0$ such that $R\left(Y_{t}\right) \subset[-M(t), M(t)]$ and the sequence $R\left(Y_{t}\right)$ is increasing in the sense that for all $0<s \leqslant t$ we have $R\left(Y_{s}\right) \subseteq R\left(Y_{t}\right)$, we say that $\mathcal{S}$ is the cone associated to the stochastic process $Y$ or simply, the cone of $Y{ }^{2}$

\footnotetext{
${ }^{2}$ Observe that in the definition, the cone of $Y$ is defined for $t>0$; this is because the range of $Y_{t}$ is trivially equal to the constant $Y_{0}$ at $t=0$.
} 
Note that the concept of range envelope differs from the notion of envelope in two points Veneziano (1979). First, the latter is a set-valued random process which upperbounds $\left|Y_{t}\right|$ with probability one during any finite length of time, while the former is a deterministic time-indexed sequence of compact sets.

Second, the range envelope (if it exists) is unique since it corresponds at any time $t$ to the smallest set to which $Y_{t}$ belongs with probability 1 .

Definition 4.4 (Conic process). A stochastic process is said to be conic if its range envelope is a cone. When the stochastic process is a martingale, we say that it is a conic martingale.

In this context, the word "conic" refers to the fact that the range of the process is bounded and is non-decreasing with time, so that the upper (resp. lower) bound of $Y_{t}$ increases (resp. decreases) with $t$.

Notice that only processes that are bounded up to $T$ admit a cone. For instance, neither Brownian motion nor its Doléans-Dade exponential are conic processes in the sense of the above definitions. In the one dimensional case, any martingale evolving between two deterministic real-valued functions of time $a(t), b(t)$ satisfying $-M<a(t) \leqslant b(t)<M$ for all $t \in \mathbb{R}_{+}$admits a cone.

The next corollary shows that conic and locally bounded martingales are in fact a same thing.

Corollary 4.1. Any conic martingale is a locally bounded martingale. Reciprocally, any locally bounded continuous martingale is a conic martingale.

Note that on $[0, T]$, conic martingales $X$ are martingales such that $X_{T}$ is bounded.

Proof. The first assertion is obvious: indeed, by definition, a conic martingale $Y$ is a martingale evolving between bounds $-\infty<a(t) \leqslant Y_{t} \leqslant b(t)<\infty$; it is therefore locally bounded. Let us prove the converse. Let $Y$ be a martingale such that for all $t>0$ there exists $a(t), b(t) \in \mathbb{R}: a(t) \leqslant Y_{t} \leqslant b(t)$. Because the paths of $Y$ are almost surely continuous, the support of $Y_{t}$ cannot have "holes" so that $R\left(Y_{t}\right)=[a(t), b(t)]$ are closed intervals. It remains to prove that the time-indexed sequence of ranges is increasing. To that end, suppose there exists $y_{s} \in R\left(Y_{s}\right)$ that does not belong to $R\left(Y_{t}\right)$ for some $t>s$. Then, either $y_{s}>\sup R\left(Y_{t}\right)$ or $y_{s}<\inf R\left(Y_{t}\right)$. In both cases, $\mathbb{E}\left[Y_{t} \mid \mathcal{F}_{s}\right] \neq y_{s}$ as $Y_{t}>y_{s}$ or $Y_{t}<y_{s}$ with probability one, respectively; $Y$ fails to be a martingale. Consequently, a necessary condition for $Y$ to be a martingale is that the range of $Y$ is an increasing sequence.

In order for the solution $Y$ of a driftless $\mathrm{SDE}$ to exist, we must have $\int_{0}^{t} \sigma^{2}\left(s, Y_{s}\right) d s<\infty$ almost surely. It is then a local martingale, see e.g. Karatzas and Shreve (2005). The following well-known result (Th. 5.1 in Protter (2005)) fills the gap between local and genuine martingales in the special case of (globally) bounded processes. 
Theorem 4.1. Every bounded local martingale is a martingale.

In the sequel, we shall focus on separable diffusion coefficients, defined below.

Definition 4.5 (Separable diffusion coefficient). A diffusion coefficient $\sigma(t, x)$ is said separable if it can be written as

$$
\sigma(t, x)=g(t) h(x)
$$

with $h$ continuous.

It is obvious that when the diffusion coefficient is separable where the time component $g$ is a bounded function for all $t \geqslant 0$, the solution $Y$ to $\operatorname{SDE}(2.1)$ with initial condition $Y_{0} \in[a, b]$ is a continuous martingale in $[a, b]$ provided that function $h(x)$ is continuous on $[a, b]$ and satisfies $h(x)>0$ for $x \in(a, b)$ and $h(x)=0$ for $x \in\{a, b\}$.

Obviously, analysis of martingales with constant cone can be restricted to martingales with standard cone $[0,1]$ as any martingale in $[a, b]$ can be obtained from a martingale in $[0,1]$. We thus have the following corollary.

Corollary 4.2. Consider the separable diffusion coefficient in eq.(4.1) where $g(t)>0, h(x)>0$ for $x \in] a, b\left[\right.$ and $h(x)=0$ elsewhere. Then, if $S D E$ (2.1) admits a unique solution $Y$ satisfying $\left.Y_{0} \in\right] a, b[$, $Y \in[a, b]$. It is therefore a bounded local martingale, and from Theorem 4.1, a genuine martingale.

Proof. The SDE $d Y_{t}=g(t) h\left(Y_{t}\right) \mathbb{I}_{\left\{Y_{t} \in[a, b]\right\}} d W_{t}$ admits a unique solution, which is a Markov process. Denote by $\tau$ the first hitting time of the boundary. In the case $\tau<\infty$, one obvious solution $Y$ on $[\tau, \infty)$ given $Y_{\tau}$ is $Y_{t}=Y_{\tau} \in\{a, b\}$. Therefore, the solution $Y$ to the above SDE is unique and belongs to $[a, b]$. Because $Y \in[a, b] \mathbb{Q}$-a.s. and $h(a)=h(b)=0$, the solution to $d Y_{t}=g(t) h\left(Y_{t}\right) \mathbb{I}_{\left\{Y_{t} \in[a, b]\right\}} d W_{t}$ is the same as that of $d Y_{t}=g(t) h\left(Y_{t}\right) d W_{t}$.

\section{Construction of conic martingales}

Obviously, any martingale with cone in $[0,1]$ is of the form $\mathbb{E}\left[\zeta \mid \mathcal{F}_{t}\right]$ for a random variable $\zeta$, valued in $[0,1]$. However, it is not possible to compute the diffusion coefficient of such martingales, since these martingales are not always diffusion processes.

The above section shows how martingales evolving in the compact set $[a, b]$ can be obtained, by adequately choosing the diffusion term $\sigma(t, x)$ in eq. (2.1). This framework is interesting theoretically but may be hard to deal with in practice. To illustrate this, suppose we want to construct a martingale 
in $[0,1]$. To this end, we can choose $Y_{0} \in[0,1], \sigma(t, x)=g(t) h(x)>0$ for all $t>0$ and $x \in(0,1)$ where $h=0$ for $x \notin(0,1)$. One simple "smooth function" satisfying the required conditions is

$$
\sigma(t, x)=\eta x(1-x)
$$

where $\eta$ is a constant, which is proven to satisfy the conditions of Theorem 3.1, and $Y \in[0,1]$ if $Y_{0} \in[0,1] .^{3}$ This leads to the SDE:

$$
d Y_{t}=\eta Y_{t}\left(1-Y_{t}\right) d W_{t}, \quad Y_{0} \in[0,1]
$$

Theorem 3.1 ensures that this SDE admits a solution which, from Corollary 4.2, is a martingale. Moreover, because $\sigma(t, 0)=\sigma(t, 1)=0$ and $\sigma(t, x)>0$ for $x \in(0,1)$, the range of this process is $[0,1]$ provided that $Y_{0} \in[0,1]$; it is thus a conic martingale with cone $[0,1]$, as per Definition 4.4. However, even if numerical schemes can be worked out to estimate the distribution of $Y_{t}$, the analytical expression of such atypical SDE may not be trivial to find, if existing. Moreover, from a practical perspective, such schemes need to guarantee that all paths (for Monte-Carlo simulation, or the range of the distribution, for PDE solver) of $Y$ remains in the cone associated to the theoretical solution (that one may guess from the SDE). Generally speaking, implicit schemes satisfying the boundedness conditions are required, but can be tedious to find out.

To address these two issues, we propose a specific construction scheme that yields the conic martingale $Y$ as a transformation of a simpler (unconstrained, or "free") process $X$ via some smooth functional $Y_{t}=F\left(t, X_{t}\right)$. The SDE and statistics of $Y$ can thus directly be obtained through those of $X$.

Here below we first show how one can create one-dimensional martingale with cone $\mathcal{S}=[0,1]$.

\subsection{Methodology}

We now proceed with the next result, which is an important tool for constructing conic martingales.

We shall need conditions ensuring existence and uniqueness of solutions to generic SDE of the form

$$
d X_{t}=\mu\left(t, X_{t}\right) d t+\eta\left(t, X_{t}\right) d W_{t}
$$

which can be found (see, e.g., Øksendal (2003),Kloeden and Platen (1999), (Jeanblanc et al., 2007, Sections 1.5.4 and 1.5.1), (Revuz and Yor, 1999, Chapter IV, Section 3)).

\footnotetext{
${ }^{3}$ In the sequel, we shall deal with processes that belong to $[0,1]$ almost surely. In that case, we shall restrict ourselves to specify the diffusion coefficient $\sigma(t, x)$ on $\mathbb{R}^{+} \times[0,1]$. Of course, the later can trivially be extended to $x \in \mathbb{R}$ via the indicator function $\mathbb{I}_{\{x \in(0,1)\}}$. This preserves the dynamics of the process and allows us to rely on existence and uniqueness results, which require the SDE coefficients to be defined for $x \in \mathbb{R}$; see e.g. proof of Corollary 4.2
} 
Theorem 5.1 (Autonomous Mapped Martingales). Let $F(x): \operatorname{dom}(F) \rightarrow[0,1], x \mapsto F(x)$ be a strictly monotonic function of class $\mathcal{C}^{2}$ with bounded first derivative. Note $f(x):=F^{\prime}(x)$ and $f^{\prime}(x)=F^{\prime \prime}(x)$. Let $\eta$ be a function defined on $\mathbb{R}^{+} \times \operatorname{dom}(F)$. Assume that there exists a process $X$ with $R\left(X_{t}\right) \in \operatorname{dom}(F)$ solution of the stochastic differential equation (SDE)

$$
d X_{t}=\frac{\eta^{2}\left(t, X_{t}\right)}{2} \psi\left(X_{t}\right) d t+\eta\left(t, X_{t}\right) d W_{t}
$$

where $\psi(x):=-\frac{f^{\prime}(x)}{f(x)}$ is the score function associated to $F$. Then, the process

$$
Y_{t}=F\left(X_{t}\right), \quad t \geqslant 0
$$

is a martingale in $[0,1]$. If the range of $X$ coincides with $\operatorname{dom}(F)$, the range of $Y$ is $[0,1]$. In this case, $Y$ is called a conic martingale with cone $[0,1]$ or equivalently, a $[0,1]$-martingale.

Proof. The process $Y=F(Z)$, valued in $[0,1]$, has dynamics given via Itô's lemma:

$$
d Y_{t}=f\left(Z_{t}\right) \eta\left(t, Z_{t}\right) d W_{t}
$$

The process $Y$ is then a bounded local martingale hence a martingale.

Notice that since $F$ is a bijection, it is invertible, and the dynamics for $Y$ becomes

$$
d Y_{t}=f \circ F^{-1}\left(Y_{t}\right) \eta\left(t, F^{-1}\left(Y_{t}\right)\right) d W_{t}=: \sigma\left(t, Y_{t}\right) d W_{t}
$$

so that $Y$ is a diffusion.

Corollary 5.1. Let $F$ be a bijection of class $\mathcal{C}^{2}$ and $X$ be the solution to eq. (5.3). Then $Y=F(X)$ is a conic martingale with cone $[0,1]$ satisfying the SDE (5.4). Moreover, the cumulative distribution function of $Y_{t}\left(F_{Y_{t}}(y)\right)$ can be obtained form that of the latent random variable $X_{t}$. In particular, if $F$ is increasing:

$$
F_{Y_{t}}(y)=F_{X_{t}}\left(F^{-1}(y)\right)
$$

Remark 5.1. Simple candidates for function $F$ are cumulative distribution (or survival distribution) functions defined on the real line, admitting a continuously differentiable density and invertible.

\subsection{Examples}

1. Let $F(x)=e^{-\lambda x}$ and $\eta(t, x)=\eta x$. Then $X$ satisfies a variant of the Verhulst equation:

$$
d X_{t}=\lambda\left(\eta^{2} / 2\right) X_{t}^{2} d t+\eta X_{t} d W_{t}
$$


with solution

$$
X_{t}=\frac{\Theta_{t}}{1-\left(\lambda \eta^{2} / 2\right) \int_{0}^{t} \Theta_{s} d s}, \quad \Theta_{t}:=X_{0} \mathrm{e}^{-\left(\eta^{2} / 2\right) t+\eta W_{t}}
$$

up to explosion time $\tau:=\inf \left\{t: \int_{0}^{t} \Theta_{s} d s=2 / \lambda \eta^{2}\right\}$.

The process $Y$ defined as $Y_{t}=\exp \left(-\lambda X_{t}\right), t \leqslant \tau$ defines a martingale (valued in $[0,1]$ ) with SDE given by

$$
d Y_{t}=-\eta \ln \left(Y_{t}\right) Y_{t} d W_{t}
$$

Note that, by construction, $\inf \left\{t: Y_{t} \in\{0,1\}\right\}=\inf \left\{t: Y_{t}=0\right\}=: \tau$. The boundary 1 is not reached (up to $\tau$ ) and $Y_{\tau \vee t}=0$.

2. Let us come back to the $\operatorname{SDE}$ (5.1). We can see that it consists in eq. (5.4) with $\sigma(t, x)=\eta x(1-x)$. Setting $\eta(t, x)=\eta$ in (5.3), it appears that we must have $f\left(F^{-1}(y)\right)=y(1-y)$. Changing the variable $x=F^{-1}(y)$ leads to the (logistic) first-order non-linear differential equation

$$
f(x)=\frac{d F(x)}{d x}=F(x)(1-F(x))
$$

which solution is proven to be

$$
F(x)=\frac{c \mathrm{e}^{x}}{1+c \mathrm{e}^{x}}, \quad \psi(x)=2 F(x)-1
$$

This function is the cumulative distribution function of a logistic random variable with mean $-\ln (c)$ and variance $\pi^{2} / 3 .{ }^{4}$ In other words, it appears that the conic martingale process $Y$ defined by eq. (5.1) can be obtained by simply mapping through the above (distribution) function $F$ the unconstrained (latent) process $X$ (defined by the SDE (5.3)) which instantaneous variance is set constant $\eta^{2}(t, x)=\eta^{2}$ and the drift, as per Theorem 5.1, given by $\frac{\eta^{2}}{2} \psi(x)$ where $\psi(x)=-\frac{f^{\prime}(x)}{f(x)}=$ $2 F(x)-1=\tanh (x / 2)$ is the score function associated to the above distribution function. In the specific case where $c=1$, the $\mathrm{SDE}$ of the latent process writes

$$
d X_{t}=\frac{\eta^{2}}{2} \tanh \left(\frac{X_{t}}{2}\right) d t+\eta d W_{t}
$$

The coefficients of this SDE satisfy the usual conditions ensuring existence and uniqueness of a solution $X$, so that the solution $Y$ to $\operatorname{SDE}(5.8)$ exists and is unique, too.

3. It is obvious that a same SDE for $Y=F(X)$ can be obtained from various combinations of $(X, F)$. In the above example, $c$ can be chosen to be any positive scalar, but provided that the correct drift (score function) is used for $X$, the same SDE is obtained for $Y$. Similarly, a given latent process

${ }^{4}$ Note that since $c \mathrm{e}^{X_{0}}=F\left(X_{0}\right) /\left(1-F\left(X_{0}\right)\right)$ and $\operatorname{dom}(F)=[0,1]$, we must have $c>0$. 
$X$ can lead to several driftless equations for $Y=F(X)$, depending on the choice of $F$. This results from the fact that different mappings $F$ can lead to the same score function. Therefore, for a given drifted process $X$, one can find several mappings $F$ such that the resulting SDE of $Y=F(X)$ is driftless. For instance, setting $F(x)=\tanh (x / 2)$ leads to $\sigma(t, y)=\frac{\eta}{2}\left(1-y^{2}\right)$ :

$$
d Y_{t}=\frac{\eta}{2}\left(1-Y_{t}^{2}\right) d W_{t}
$$

The score function of $F(x)=\tanh (x / 2)$ is equal to $\tanh (x / 2)$ as well, which is the same as that of $F(x)=\mathrm{e}^{x} /\left(1+\mathrm{e}^{x}\right)$ that led to $\operatorname{SDE}(5 \cdot 1)$.

4. Consider the standard Gaussian case, $F(x)=\Phi(x)$. The score function of the Gaussian distribution is given by $\psi(x)=-\phi^{\prime}(x) / \phi(x)=x$ where $\phi(x)$ is the standard Normal density function, and we get that $Y=\Phi(X)$ is a $[0,1]$-martingale provided that the $\mathrm{SDE}$

$$
d X_{t}=\frac{\eta^{2}\left(t, X_{t}\right)}{2} X_{t} d t+\eta\left(t, X_{t}\right) d W_{t}
$$

has a solution and $Y$ has diffusion coefficient

$$
\sigma(t, y)=\phi \circ \Phi^{-1}(y) \eta\left(t, \Phi^{-1}(y)\right)
$$

\subsection{Another conic martingale}

The standard Normal distribution function $\Phi$ can be used to turn any Itô integral into a martingale in $[0,1]$ with given initial value $x \in(0,1)$.

Define

$$
Z_{t}:=z+\int_{0}^{t} \sigma_{s} d W_{s}
$$

where $z \in \mathbb{R}$ and $\sigma$ is adapted to the natural filtration of $W$.

Lemma 5.1. Let $x \in(0,1)$ and set $z:=\Phi^{-1}(x)$. For $\Upsilon:=1 / \sqrt{1-[Z]}$, setting $\tau=\inf \left\{t:[Z]_{t}=1\right\}$, the stochastic process $\mathcal{M}(Z)$ defined as

$$
\mathcal{M}(Z)_{t}:=\Phi\left(\Upsilon_{t} Z_{t}\right), t<\tau
$$

is a martingale in $[0,1]$ with initial value $x$. Moreover, if $R\left(X_{t}\right)=\mathbb{R}$, then $\mathcal{M}(Z)_{t}$ is a $[0,1]$-martingale.

Proof. It is obvious to see that $\mathcal{M}(Z) \in[0,1]$ and that $\mathcal{M}(Z)_{0}=x$ since $\Upsilon_{0}=1$ and $Z_{0}=z$. From Theorem 4.1, it remains to show that it is a local martingale. This is straightforward using the property $\Phi^{\prime \prime}(x)=-x \Phi^{\prime}(x)=:-x \phi(x)$ and the fact that $d \Upsilon_{t}=\frac{\Upsilon_{t}^{3}}{2} d[Z]_{t}$. Indeed, from Itô's lemma,

$$
\begin{aligned}
\frac{d \mathcal{M}(Z)_{t}}{\phi \circ \Phi^{-1}\left(\mathcal{M}(Z)_{t}\right)} & =Z_{t} d \Upsilon_{t}+\Upsilon_{t} d Z_{t}-\frac{\Upsilon_{t} Z_{t}}{2} \Upsilon_{t}^{2} d[Z]_{t} \\
& =\Upsilon_{t} d Z_{t}
\end{aligned}
$$


The mapping $\mathcal{M}$ turns any continuous local martingale $Z$ defined on $\mathbb{R}$ into a [0,1]-martingale $\mathcal{M}(Z)_{t}=\Phi\left(Z_{t} / \sqrt{1-[Z]_{t}}\right)$ using function $\Phi$. This is similar to the Doléans-Dade exponential $\mathcal{E}$, which maps $Z$ to a non-negative martingale $\mathcal{E}(Z)_{t}=\exp \left\{Z_{t}-[Z]_{t} / 2\right\}$ using the exponential function provided that $Z$ satisfies the Novikov condition. This results from the connections between first and second derivatives of these functions. From this perspective, the martingale $\mathcal{M}(Z)$ can be seen as the equivalent of $\mathcal{E}(Z)$ but for $[0,1]$-martingales instead of $\mathbb{R}^{+}$-martingales.

Remark 5.2. An important point is that the process $\mathcal{M}(Z)$ reaches the bounds if and only if $[Z]$ can reach 1. Assume for instance a constant diffusion coefficient $\sigma_{t}=\eta \in \mathbb{R}_{0}^{+}$. Then, $[Z]_{t}=\eta^{2} t$ so that $\frac{Z_{t}}{\sqrt{1-[Z]_{t}}}$ is a.s. finite for $t<\eta^{-2}$ but $\mathcal{M}(Z)_{\eta^{-2}} \in\{0,1\}$ a.s., i.e. we reach (and stick to) one of the boundaries at $t=1 / \eta^{2}$. On the other hand, $\mathcal{M}(Z)$ cannot reach the boundaries if we choose $\sigma_{t}=\sigma(t)=\eta e^{-\eta^{2} t / 2}$ since $\int_{0}^{\infty} \sigma_{s}^{2} d s=1$. Moreover, the process $\mathcal{M}(Z)$ is a diffusion if $\sigma_{t}=\sigma(t)$ is a deterministic function of time. In that case, the diffusion coefficient of $\mathcal{M}(Z)$ is separable in the sense of eq. (4.1) with $g(t)=\sigma(t) \Upsilon_{t}=\sigma(t) / \sqrt{1-\int_{0}^{t} \sigma^{2}(s) d s}$ and $h(x)=\phi \circ \Phi^{-1}(x)$.

\subsection{Practical considerations}

As explained above, conic martingales can be obtained by specifying the form of the diffusion coefficient $\sigma(t, y)$. However, the resulting SDE's are most often not analytically tractable, so that numerical schemes need to be used.

The above considerations suggest that it may be better to first (analytically or numerically) try to solve the SDE of an underlying free process $X=G(Y)$, and then get the solution of $Y$ via the mapping $F=G^{-1}$. If $G$ is chosen in a clever way, it could be that the second SDE is more easy to deal with (more standard, evolving in $\mathbb{R}$ instead of e.g. $[0,1]$ ). At least we can get the correct range. This is the purpose of the next theorem, which tells us how to choose $G=F^{-1}$ so that $X_{t}=G\left(Y_{t}\right)$ takes a specific, more appealing form. More specifically, the developed methodology allows us to write the solution (if it exists) of the SDE (2.1) when the instantaneous volatility is separable (in the sense of eq. (4.1)) as a mapping $F$ of another process with specific drift but diffusion coefficient $g(t)$ depending on time only. The below theorem states sufficient conditions for the solution $Y$ to the $\mathrm{SDE}(2.1)$ to be given by $F(X)$ where $X$ is as desired. Moreover, (i) we are told which $F$ we have to choose in order for $X_{t}=F^{-1}\left(Y_{t}\right)$ to have the required dynamics, and (ii) the SDE of $X$ is completely specified.

Theorem 5.2. Consider the SDE (2.1) where the diffusion coefficient is separable in the sense of (4.1). 
Assume function g satisfies $g^{2}(t)<\infty$ for all $t$ and $h(y)$ is strictly positive of class $\mathcal{C}^{2}$ in the set $\mathcal{A} \backslash \partial \mathcal{A}$ and vanishes at the (existing) boundaries $\partial \mathcal{A}$ of $\mathcal{A}$. Let $y=F(x) \subseteq \mathcal{A}$ solve the first order autonomous non-linear $O D E^{5}$

$$
\frac{d y}{d x}=h(y)
$$

If $\psi(x)=-F^{\prime \prime}(x) / F(x)$ is Lipschitz continuous, then (2.1) admits the strong pathwise unique solution $Y_{t}=F\left(X_{t}\right)$ where $X$ is the unique strong solution to the SDE (5.3) with initial value $X_{0}:=F^{-1}\left(Y_{0}\right)$, diffusion coefficient $\eta(t, x)=g(t)$ and drift $\mu(t, x)=-\frac{g^{2}(t) F^{\prime \prime}(x)}{2 F^{\prime}(x)}=\frac{g^{2}(t)}{2} \psi(x)$.

Proof. Let us first prove that the solution $y=F(x)$ to the non-linear differential equation (5.14) is invertible, of class $\mathcal{C}^{2}$. Because $h(x)>0$ for all $x \in \mathcal{A}$ and $\operatorname{im}(F) \subseteq \operatorname{dom}(h), F(x)=\int_{-\infty}^{x} h(F(u)) d u+k$ is continuously (strictly) increasing; $F(x)$ is therefore invertible, and continuous. Moreover, from the smoothness conditions on $h$, the first three derivatives of $F$ are continuous: $F^{\prime}(x)=f(x)=h(F(x))$, $f^{\prime}(x)=h^{\prime}(F(x)) f(x)=h^{\prime}(F(x)) h(F(x))$ and $f^{\prime \prime}(x)=h^{\prime \prime}(F(x)) h^{2}(F(x))+h^{\prime 2}(F(x)) h(F(x))$. Therefore, the solution to the above ODE has the functional form $h(x)=f\left(F^{-1}(x)\right)$, where $F$ has the required smoothness for Itô's lemma to be used, and is invertible. Itô's lemma yields the dynamics of $F^{-1}\left(Y_{t}\right)$, which corresponds to the $\operatorname{SDE}(5.3)$ where $\eta(t, x)=g(t)$ and $\mu(t, x)=-\frac{g^{2}(t)}{2} \frac{f^{\prime}(x)}{f(x)}$. From Theorem 4.5.3 of Kloeden and Platen (1999) (p. 131) this SDE, with finite initial value $X_{0}=F^{-1}\left(Y_{0}\right)$ has a strong pathwise unique solution since the coefficients meet the standard requirements (Lipschitz continuity of $\psi(x)$ together with the boundedness of $g^{2}(t)$ for $t<\infty$ implies the linear growth bound condition on $g^{2}(t) \psi(x)$ and hence so is the drift coefficient $\left.\mu(t, x)\right)$. Finally, the solution $Y$ is given by the mapping $F: Y=F(X)$.

Example 5.1. Consider the case of the exponential martingale with time dependent volatility, with SDE $d Y_{t}=\eta(t) Y_{t} d W_{t}$. Setting $g(t)=\eta(t)$ and $h(x)=x$, we find $F(x)=e^{x}+k ; \mu(t, x)=-\eta(t)^{2} / 2$. In the case where $\eta(t)=\eta$, one could equivalently choose $g(t)=1$ and $h(x)=\sigma x$, in which case $F(x)=e^{\eta x}+k$ and $\mu(t, x)=-\eta / 2$.

Example 5.2. This trick has been previously applied to the SDE (5.1) in the case $g(t)=\eta$ and $h(x)=$ $x(1-x)$. Similarly regarding eq. (5.10), we can set $g(t)=\eta$ and $h(x)=\left(1-x^{2}\right) / 2$; the solution to the ODE (5.14) leads to $F(x)=\tanh (x / 2)$; therefore, the solution to (5.10) is given by $Y=\tanh (X / 2)$ where $X$ is the solution to (5.3).

\footnotetext{
${ }^{5}$ The solution to this ODE is proven to have the general form $y(x)=H(x+k)$ where $k$ is the integration constant and $H(x)$ is the inverse of $\int_{\inf \operatorname{dom}(h)}^{x} \frac{1}{h(u)} d u$
} 
Remark 5.3. It is worth noting that although we obtain the SDE of $X=F^{-1}(Y)$ from that of $Y$, the expression of $F^{-1}$ is not needed; it does not enter the SDE of $X$. The drift of $X$ is determined by the score function of $F$, which solves the ODE.

\section{The $\Phi$-martingale}

The previous computations done in eq. (5.11) for $F=\Phi$ lead, for $\eta(t, x)=\eta$ to $d X_{t}=\frac{\eta^{2}}{2} X_{t} d t+\eta d W_{t}$, i.e. $X$ is a Vasicek process

$$
X_{t}=X_{0} \mathrm{e}^{\frac{\eta^{2}}{2} t}+\eta \mathrm{e}^{\frac{\eta^{2}}{2} t} \int_{0}^{t} \mathrm{e}^{-\frac{\eta^{2}}{2} s} d W_{s}
$$

with constant diffusion coefficient $\eta$, zero long-term mean and negative speed of mean reversion $\eta^{2} / 2$. Note that, for fixed $t, X_{t}$ has the same law as

$$
X_{0} \mathrm{e}^{\frac{\eta^{2}}{2} t}+\sqrt{e^{\eta^{2} t}-1} Z
$$

where $Z$ is a standard Gaussian random variable. This leads to a $[0,1]$-martingale $Y$ which analytical expression is $\Phi(X)$ where $X=\left(X_{t}\right)_{t \geqslant 0}$ is the Vasicek process (6.1). The process $Y$ is called the $\Phi$ martingale. Sample paths drawn from this exact solution are shown in Fig. 1.

Remark 6.1. It has been shown that the case (5.8) is also tractable since there is closed form expression for the latent process $X$ (and thus for $Y=\exp (-\lambda X)$ ). The joint density of $\left(\Theta_{t}, \int_{0}^{t} \Theta_{s} d s\right)$ has been studied by Yor in (Yor, 1992), providing the law of $X_{t}$ (see Appendix). Howhever, the solution $X_{t}$

explodes at $\tau=\left\{t: \int_{0}^{t} \Theta_{s} d s=2 /\left(\lambda \eta^{2}\right)\right\}$, and $\tau<\infty$ wp 1 as $\Theta_{s}$ is a grounded positive process. This means that the corresponding $Y_{t}=e^{-\lambda X_{t}}, \lambda>0$, will collapse to zero in finite time as well. This is not the case of the $\Phi$-martingale which merely asymptotically collapses to the bounds, but belongs to $(0,1)$ $\mathbb{Q}$-a.s. for all $t>0$ (see Section 6.1.2).

\subsection{Statistics and asymptotics}

In the case where the SDE of the latent process $X$ (which drift is implied by $F$ ) has an explicit solution, the process $Y$ can be studied in details. For instance, the asymptotic distribution of $Y_{t}$ as $t \rightarrow \infty$ can be obtained. Moreover, one can also study the properties of disjoint increments of $Y$. They have zero-mean and are uncorrelated, as per the martingale property. Their variances and quantile functions can be computed as well. We study below the statistics of the $\Phi$-martingale. For the sake of comparison, we mention the corresponding results for the exponential martingale. 


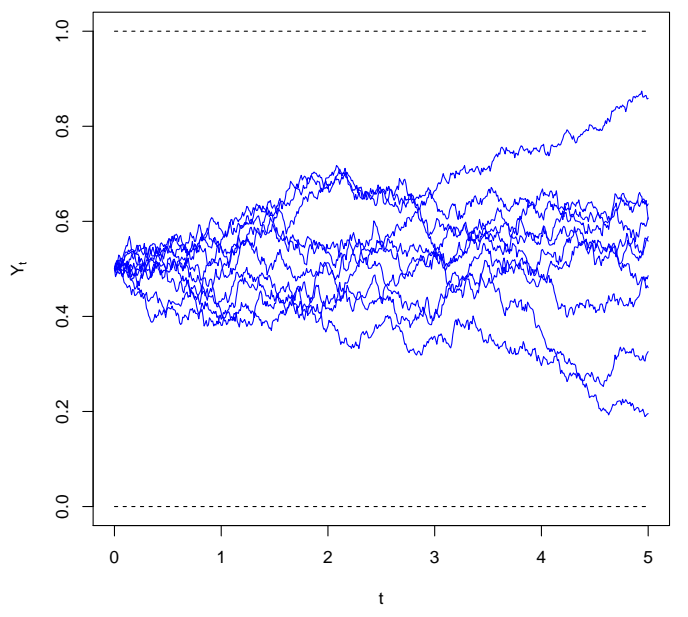

(a) $\left(Y_{0}, \eta\right)=(0.5,0.2)$

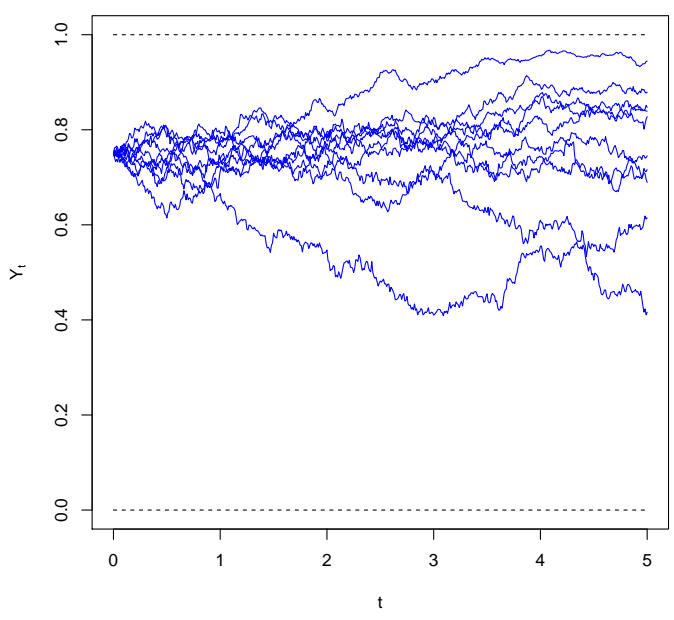

(c) $\left(Y_{0}, \eta\right)=(0.75,0.2)$

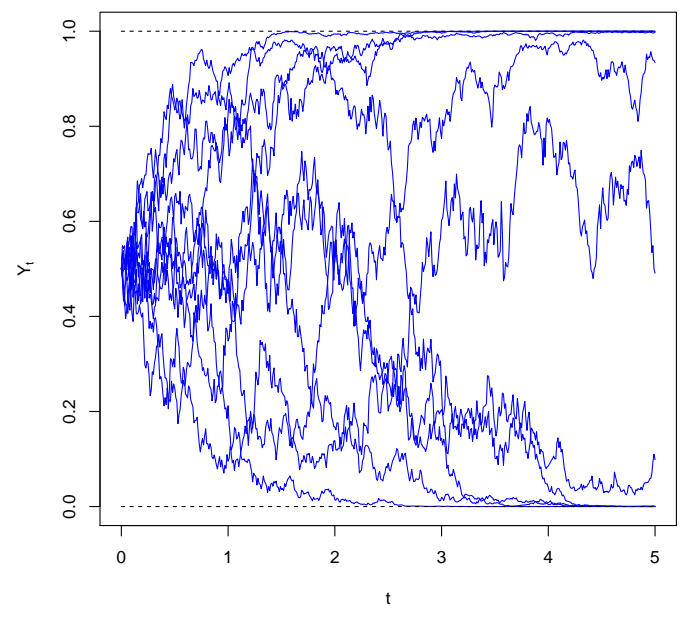

(b) $\left(Y_{0}, \eta\right)=(0.5,0.8)$

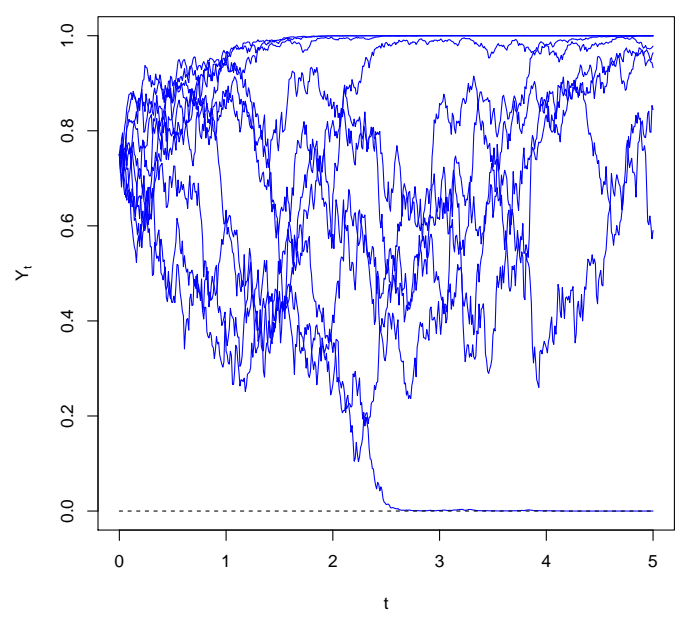

(d) $\left(Y_{0}, \eta\right)=(0.75,0.8)$

Figure 1: Ten sample paths of the $\Phi$-martingale $Y$ drawn from the exact solution $\Phi(X)$ for various diffusion scales $\eta$ and initial conditions $Y_{0}$. 
In the case of $Y=\Phi(X)$ where $X$ is a Vasicek process with instantaneous variance $\eta^{2}$, zero longterm mean and negative mean reversion speed $\eta^{2} / 2$, the variance of the random variable $Y_{t}$ is given by $\mathbb{E}\left[Y_{t}^{2}\right]-Y_{0}^{2}$ where

$$
\begin{aligned}
\mathbb{E}\left[Y_{t}^{2}\right] & =\int_{0}^{1} \frac{y^{2}}{f\left(F^{-1}(y)\right)} f_{X_{t}}\left(F^{-1}(y)\right) d y \\
& =\Phi_{2}\left(X_{0}, X_{0} ; \frac{\mathrm{e}^{\eta^{2} t}-1}{\mathrm{e}^{\eta^{2} t}}\right) \\
& =\Phi_{2}\left(X_{0}, X_{0} ; 1-\mathrm{e}^{-\eta^{2} t}\right)
\end{aligned}
$$

where $\Phi_{2}(x, y ; \rho)$ is the standard bivariate Normal cumulative distribution with correlation $\rho$. In particular, $\lim _{t \rightarrow \infty} \mathbb{E}\left[Y_{t}^{2}\right]-Y_{0}^{2}=\Phi\left(X_{0}\right)-Y_{0}^{2}=Y_{0}\left(1-Y_{0}\right)$.

As per properties of martingales $\mathbb{E}\left[Y_{s} Y_{t}\right]=\mathbb{E}\left[Y_{s \wedge t}^{2}\right]$ so that for any $\delta \geqslant 0$, the auto-covariance of $\left\{Y_{t}, Y_{t+\delta}\right\}$ is equal to the variance of $Y_{t}$. The variance of the increments is then given by

$$
\begin{aligned}
\operatorname{var}\left[Y_{t+\delta}-Y_{t}\right] & =\operatorname{var}\left[Y_{t+\delta}\right]-\operatorname{var}\left[Y_{t}\right] \\
& =\mathbb{E}\left[Y_{t+\delta}^{2}\right]-\mathbb{E}\left[Y_{t}^{2}\right] \\
& =\Phi_{2}\left(X_{0}, X_{0} ; 1-\mathrm{e}^{-\eta^{2}(t+\delta)}\right)-\Phi_{2}\left(X_{0}, X_{0} ; 1-\mathrm{e}^{-\eta^{2} t}\right)
\end{aligned}
$$

which converges to zero as $t \rightarrow \infty$. Intuitively, this means that the "activity" of the process (path by path) will decrease with time, and the process will converge to some constant level. By comparison, the variance of the exponential martingale $M_{t}=M_{0} \mathrm{e}^{-\eta^{2} / 2 t+\eta W_{t}}$ increases with $t$ :

$$
\begin{aligned}
\operatorname{var}\left[M_{t}-M_{s}\right]= & \mathbb{E}\left[\left(M_{t}-M_{s}\right)^{2}\right]=\mathbb{E}\left[M_{s}^{2}\left(\frac{M_{t}}{M_{s}}-1\right)^{2}\right] \\
= & \mathbb{E}\left[M_{s}^{2}\right]\left(\mathbb{E}\left[\frac{M_{t}^{2}}{M_{s}^{2}}\right]-2 \mathbb{E}\left[\frac{M_{t}}{M_{s}}\right]+1\right) \\
= & X_{0} \mathrm{e}^{\eta^{2} s} \mathbb{E}\left[\mathrm{e}^{-(2 \eta)^{2} / 2 s+2 \eta \sqrt{s} Z}\right] \times\left(\mathrm{e}^{\eta^{2}(t-s)} \mathbb{E}\left[\mathrm{e}^{-(2 \eta)^{2} / 2(t-s)+2 \eta \sqrt{t-s} Z}\right]\right. \\
& \left.-2 \mathbb{E}\left[\mathrm{e}^{-\eta^{2} / 2(t-s)+\eta \sqrt{t-s} Z}\right]+1\right) \\
= & X_{0} \mathrm{e}^{\eta^{2} s}\left(\mathrm{e}^{\eta^{2}(t-s)}-1\right)
\end{aligned}
$$

Because the paths of the $\Phi$-martingale $Y$ evolve between two bounds, a central question is to determine whether they collapse to the bounds, in which case the distribution of $Y_{t}$ would have less and less mass in $(0,1)$ in the sense that for any arbitrarily small threshold $\epsilon>0$ and any probability level $0<p<1$, there exists a time $t_{\epsilon}$ such that for all $t>t_{\epsilon}, \mathbb{Q}\left\{Y_{t} \in[0, \epsilon) \cup(1-\epsilon, 1]\right\}>p$; $Y_{t}$ ends up in the neighborhood of the bounds with any desired confidence interval. This will be proven in the case when $F=\Phi$ and $\eta(t)=\eta$ (Section 6.1.2). An intuitive development is provided in Appendix (9.2) in the more general case of bounded martingales. 
This might be an argument to show that this specific setup is not appropriate in many cases. However, this distribution behavior is shared by the quite popular geometric Brownian motion for example. The distribution of the exponential martingale is collapsing to 0 as $t \rightarrow \infty$ (see Fig. 2 for an illustration of the quantiles for the corresponding stochastic processes $\left.M_{t}, q(t, p):=q: \mathbb{Q}\left\{M_{t} \leqslant q\right\}=p\right)$. The fact that for this process, the variance of $M_{t+\delta}-M_{t}$ is not converging to zero as time passes in spite of this collapsing feature results from the fact that the right tail of the exponential martingale distribution is unbounded.

\subsubsection{Asymptotic distribution of the exponential martingale}

Consider the exponential martingale $M$ introduced above. The corresponding quantile function $q(t, p)$ defined according to $\mathbb{Q}\left\{\left[M_{t} \leqslant q(t, p)\right\}=p\right.$ is

$$
q(t, p)=\exp \left(\eta \sqrt{t} \Phi^{-1}(p)-\eta^{2} / 2 t\right)
$$

and for $0<p<1$,

$$
\lim _{t \rightarrow \infty} q(t, p)=\lim _{t \rightarrow \infty} \exp (\sqrt{t}(a-b \sqrt{t}))
$$

for some finite $-\infty<a:=\eta \Phi^{-1}(p)<\infty$ and $0<b:=\eta^{2} / 2<\infty$. For $t \geqslant t^{\star}:=\max (0, a / b)$, the expression in the RHS limit is strictly decreasing to 0 with respect to $t$. The $p=50 \%$ case (median) is precisely the largest $p$ such that the curve is decreasing everywhere. ${ }^{6}$

\subsubsection{Asymptotic distribution of the $\Phi$-martingale $Y(\eta(t)=\eta)$}

We get

$$
\begin{aligned}
\lim _{t \rightarrow \infty} \mathbb{Q}\left\{Y_{t} \leqslant y\right\} & =\lim _{t \rightarrow \infty} \Phi\left(\Phi^{-1}(y), \Phi^{-1}\left(Y_{0}\right) \mathrm{e}^{\eta^{2} t / 2}, \sqrt{\mathrm{e}^{\eta^{2} t}-1}\right) \\
& =\lim _{t \rightarrow \infty} \Phi\left(\frac{\Phi^{-1}(y)-\Phi^{-1}\left(Y_{0}\right) \mathrm{e}^{\eta^{2} t / 2}}{\sqrt{\mathrm{e}^{\eta^{2} t}-1}}\right) \\
& =0 \mathbb{I}_{\{y=0\}}+\Phi\left(-\Phi^{-1}\left(Y_{0}\right)\right) \mathbb{I}_{\{0<y<1\}}+\mathbb{I}_{\{y=1\}} \\
& =\left(1-Y_{0}\right) \mathbb{I}_{\{0<y<1\}}+\mathbb{I}_{\{y=1\}}
\end{aligned}
$$

Therefore, $Y_{t}$ converges in distribution to Bernoulli $\left(Y_{0}\right)$ as $t \rightarrow \infty$. It is worth noting that Bernoulli $\left(Y_{0}\right)$ corresponds to the distribution in $[0,1]$ with maximum variance for a given mean $Y_{0} \in[0,1]$ (this is quite intuitive and easy to prove).

\footnotetext{
${ }^{6}$ This contradicts the naive interpretation of martingales having "no tendency to raise or fall"; the exponential martingale does have a tendency to fall since $\mathbb{Q}\left\{M_{t+\delta}<M_{t}\right\}>50 \%$ but its expectation does not $\mathbb{E}\left[M_{t+\delta}\right]=\mathbb{E}\left[M_{t}\right]$. This reflects the fact that the martingale $M$ satisfies $\lim _{t \rightarrow \infty} M_{t}=0$
} 


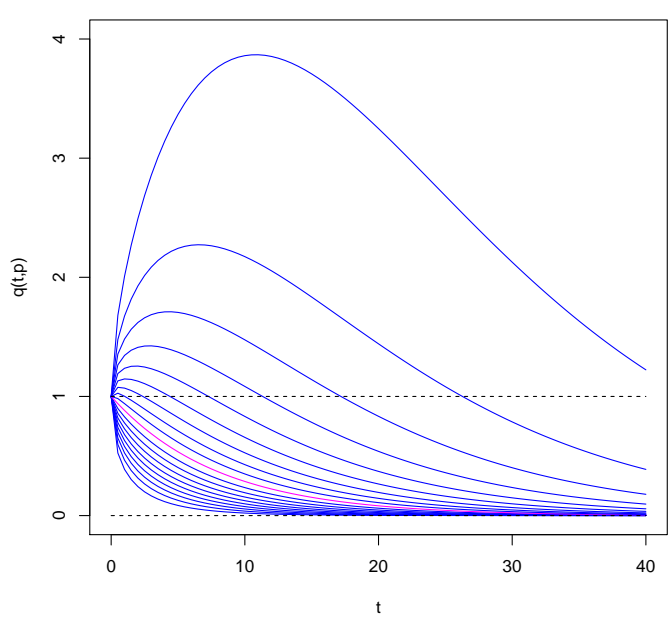

(a) Exponential martingale with $M_{0}=1$

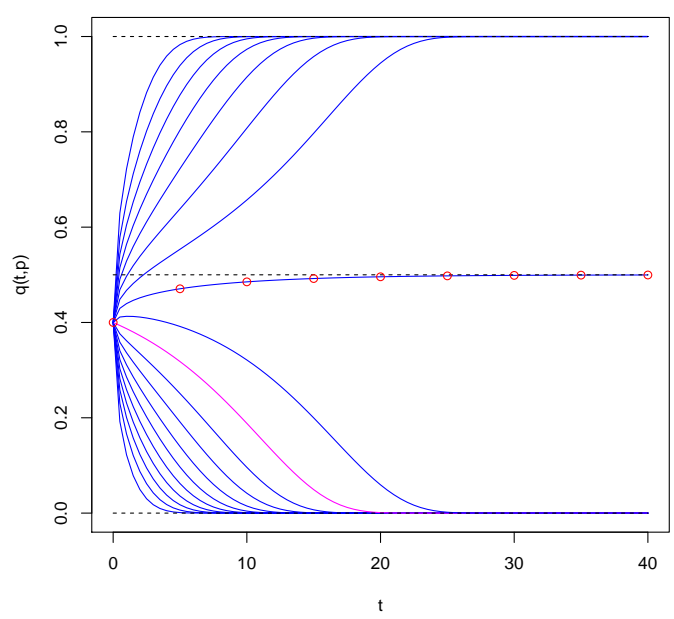

(c) $\Phi$-martingale with $Y_{0}=0.4$

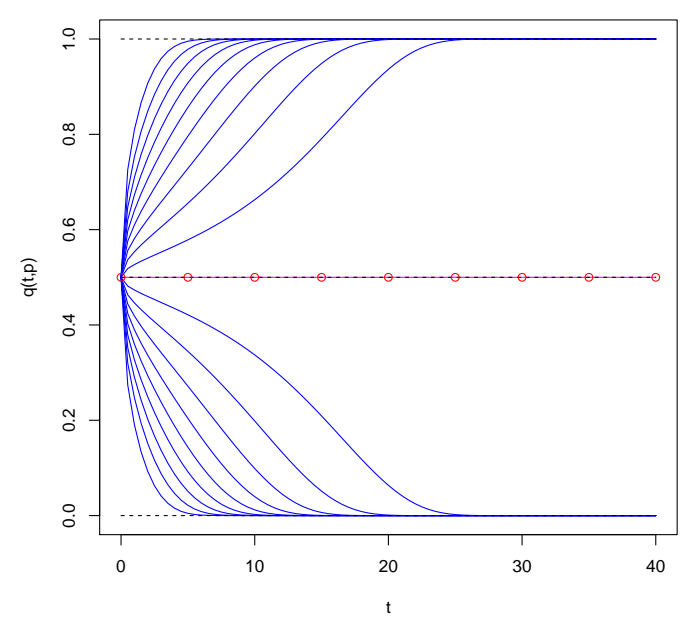

(b) $\Phi$-martingale with $Y_{0}=0.5$

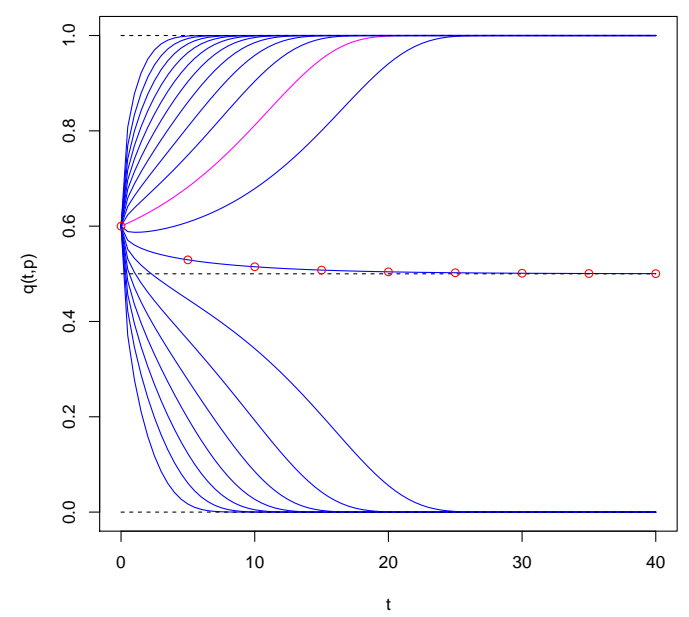

(d) $\Phi$-martingale with $Y_{0}=0.6$

Figure 2: Quantile trajectories $q(t, p)$ of the distributions of the exponential martingale $M_{t}$ and the $\Phi$-martingale $Y_{t}$ for $\eta=50 \%$. The curves are shown for $p \in\{5 \%, 10 \%, \ldots, 95 \%\}$ probability levels (of course, the corresponding trajectories are ordered bottom up). For the exponential martingale case, the curve associated to the median is the largest decreasing curve. For the bounded martingale case, the distribution collapses to a Bernoulli $\left(Y_{0}\right)$. The median is shown in magenta, and the $\left(1-Y_{0}\right)$-quantiles are emphasized with dots. If $Y_{0}=0.5$, the distribution is equally splitted to the bounds. 
Because the Gaussian solution $X$ does not explode, the collapsing feature of the $\Phi$-martingale is an asymptotic behavior: $\mathbb{Q}\left\{Y_{t} \in\{0,1\}\right\}=0$ for all $t>0$. This is in contrast with the case (5.6) with $\mu=\lambda \eta^{2} / 2>0$ where the process $Y=\exp (-\lambda X)$ has a positive probability to be strictly zero before any finite time: $\forall t>0, \mathbb{Q}\left\{Y_{t}=0\right\}>0$.

\subsection{Autonomous Gaussian martingales}

We are investigating in which case a continuous local martingale $Y$ which is a diffusion with separable diffusion coefficient can be written as the time-homogeneous (that is, autonomous) mapping $F$ of a Gaussian diffusion $X$.

Definition 6.1 (Gaussian Diffusion). A Gaussian diffusion is the unique solution $X$ to the SDE (5.2) where the drift $\mu(t, x)$ is affine in $x, \mu(t, x)=a(t)+b(t) x$ and the diffusion coefficient $\eta(t, x)$ is a function of time only, $\eta(t, x)=\gamma(t)<\infty$ for all $t$.

Observe that not all Gaussian processes are Gaussian diffusions in the sense of the above definition. For instance, the solution $X$ to the $\operatorname{SDE} d X_{t}=\operatorname{sign}\left(W_{t}\right) d W_{t}$ is not a Gaussian diffusion but is a Brownian motion (and thus a Gaussian process), and Fractional Brownian motions are Gaussian processes which are even not semi-martingales.

Definition 6.2 (Autonomous Gaussian Martingales). We say that the martingale $Y$ is autonomous Gaussian if (i) it can be obtained by mapping a Gaussian diffusion X through an autonomous mapping $F(x)$ and (ii) the diffusion coefficient is separable in the sense of (4.1).

Equating the $(d t)$ and $\left(d W_{t}\right)$ terms of the $Y \operatorname{SDE}(2.1)$ with that of the $F\left(t, X_{t}\right)$ SDE obtained using Itô, we get

$$
\begin{aligned}
& (a(t)+b(t) x) F_{x}(t, x)+\frac{\eta^{2}(t)}{2} F_{x x}(t, x) \stackrel{(d t)}{=} \quad-F_{t}(t, x) \\
& \gamma(t) F_{x}(t, x) \stackrel{\left(d W_{t}\right)}{=} \quad \eta(t) h \circ F(t, x)
\end{aligned}
$$

Using a time-homogeneous mapping yields $F(t, x)=F(x)$, implying that $\eta(t)=\gamma(t)$. The $\left(d W_{t}\right)$ equation then corresponds to eq. (5.14) and it solution yields the space component $h(y)$ of the separable diffusion coefficient $\sigma(t, y)=\gamma(t) h(y)$. In this exercise however, we are interested in the form of $F(x)$ that can be used so that $X$ is a Gaussian process and $Y=F(X)$ a martingale. Setting $G(x)=F_{x}(x)$, the $(d t)$ equation becomes

$$
\frac{\eta^{2}(t)}{2} G_{x}(x)+(a(t)+b(t) x) G(x)=0
$$


This is a first-order ODE which solution is given by

$$
\begin{aligned}
& G(t, x)=k_{1}(t) \mathrm{e}^{-\frac{2 a(t) x+b(t) x^{2}+c(t)}{\eta^{2}(t)}} \\
& F(t, x)=k_{1}(t) \int_{-\infty}^{x} \mathrm{e}^{-\frac{2 a(t) u+b(t) u^{2}+c(t)}{\eta^{2}(t)}} d u+k_{2}(t)
\end{aligned}
$$

As we considered time-independent mapping $(F(t, x)=F(x)$ for all $x)$, the ratios $a(t) / \eta^{2}(t), b(t) / \eta^{2}(t)$, $c(t) / \eta^{2}(t)$ and the integration constants $k_{1}(t), k_{2}(t)$ need all to be constant in order to get the required form for the $Y_{t}=F\left(X_{t}\right)$ SDE. We note them $a, b, c, k_{1}, k_{2}$. Clearly, the case $b<0$ can be excluded as the integral in eq. (6.5) does not converge in this case. We thus have three main cases for $(a, b, c)$ to analyze:

- $(0,0, c): F(x)=k_{1} \mathrm{e}^{-c} x+k_{2}$; the mapping is an affine function of the form $F(x)=\alpha x+\beta$.

- $(a<0,0, c): F(x)=\frac{-k_{1} \mathrm{e}^{-c}}{2 a} \mathrm{e}^{-2 a x}+k_{2}$, the mapping is a shifted exponential $F(x)=\frac{-k_{1} \mathrm{e}^{-c}}{2 a} \mathrm{e}^{-2 a x}+k_{2}=$ $\frac{\alpha}{\xi} \mathrm{e}^{\xi x}+k$ with $\xi>0$

- $(a, b>0, c): F(x)=\alpha \Phi\left(\frac{x-\beta}{\xi}\right)+k_{2}$ where $\alpha=k_{1} \sqrt{\pi / b} \mathrm{e}^{a^{2} / b-c}>0, \beta=-a / b$ and $\xi=1 / \sqrt{2 b}$. The mapping is a shifted and rescaled version of the Normal cumulative distribution function.

The above mappings are the only ones leading to null $d t$ term and separable diffusion coefficient for $Y_{t}=F\left(X_{t}\right)$ when $X_{t}$ is a Gaussian diffusion. This also specify the form of the space component $h(x)$ of the diffusion coefficient that can be obtained by mapping Gaussian processes through $F(x)$. From the $\left(d W_{t}\right)$ equation, we get respectively

- Since $a=b=0, X$ is a rescaled Brownian motion $\left(d\langle X, X\rangle_{t}=\gamma^{2}(t) d t\right)$ and $Y_{t}=\alpha X_{t}+k$ is a shifted and rescaled copy. In particular, $h(x)=h=\alpha$

- $Y$ is the exponential of a Gaussian process with shift: $F_{x}(x)=\alpha \mathrm{e}^{\xi x}$ so that $h(x)=F_{x}\left(F^{-1}(x)\right)=$ $\xi(x-k)$. Since $\xi>0$, a continuity argument shows that the process $Y$ is bounded below $k$ if $Y_{0}>k$.

- $Y$ is obtained by mapping the Gaussian process $X$ through a Normal cumulative distribution, rescaling and shifting. The obtained process is a $[k, \alpha+k]$-martingale. In this case, $h(x)=$ $\frac{\alpha}{\beta} \phi\left(\Phi^{-1}\left(\frac{x}{\alpha}\right)\right)$

We summarize these results in the theorem below.

Theorem 6.1 (Autonomous Gaussian Martingales). The only autonomous Gaussian martingales are (up to a deterministic shift and scaling coefficient) i) the trivial martingale, ii) the Brownian motion, iii) the geometric Brownian motion and iv) the $\Phi$-martingale. Interestingly, each resulting process has a specific range, namely: constant, unbounded, one-side bounded and two-sides bounded. 
This result says that if one wishes to construct a continuous local martingale $Y$ with separable diffusion coefficient $\sigma(t, y)=\eta(t) h(y)$ and evolving in a given set by mapping a Gaussian diffusion via an invertible autonomous function, there are not many alternatives: only one family of mapping per type of range. In particular, the $\Phi$-martingale $\Phi(X)$ is the only bounded continuous martingale with separable diffusion coefficient that can be obtained by mapping a Gaussian diffusion $X$ through a smooth autonomous function $F(x)$.

Note that if one relaxes the time-homogeneity and invertibility constraints, other solutions are possible. For instance, in the case $a(t)=b(t)=0$ and $\eta(t)=\eta\left(X_{t}=X_{0}+\eta W_{t}\right)$ and setting $F(t, x)=x^{2}-\eta^{2} t$, we obtain $Y_{t}=X_{t}^{2}-[X]_{t}$ which is a well known martingale (in $\left.\mathbb{R}\right)$.

In the next section, we show how these martingales can be used in credit risk modeling applications.

\section{Application to Survival Probabilities}

We adopt the credit risk modeling setup and focus on the default time $\tau$ of some reference entity. In this framework, one usually defines the filtration $\mathbb{F}$, which represents the market information excluding default observation. The enlarged filtration is obtained by including the explicit information relevant to the default event: $\mathcal{G}_{t}=\mathcal{F}_{t} \vee \sigma\left(\mathbb{I}_{\{\tau>s\}}, 0 \leqslant s \leqslant t\right)$ (with right-continuous regularisation). In Cox models for example, the stochastic intensity process $\lambda$ is $\mathbb{F}$-adapted, but conditional upon the path $\left(\lambda_{t}\right)_{t \geqslant 0}$, the occurrence of default $\mathbb{1}_{\{\tau \leqslant t\}}$ is an independent event. More generally, the latter is $\mathcal{G}_{t}$-measurable, but not $\mathcal{F}_{t}$-measurable. Literature on credit risk modeling emphasize that under some conditions, one can get rid of actual default modeling; default indicators can be replaced by stochastic default probabilities, working in the restricted filtration $\mathbb{F}$ instead of the complete filtration $\mathbb{G}$. We do not enter the details of this modeling approach, but refer the reader to Lando (2004) and Bielecki et al. (2011) for more information.

\subsection{Unconditional survival probability and Azéma supermartingale}

We now move to the modeling of the martingale $S_{t}(T), t \geqslant 0$ defined in eq. (1.1). This is useful in many circumstances, including the pricing of credit derivatives or to adjust the price of a derivatives portfolio to account for counterparty risk (credit value adjustment), see e.g Cesari et al. (2009), Brigo and Alfonsi (2005). As an illustration of the above methodology, we set $S_{t}(T):=\Phi\left(X_{t}(T)\right)$ where $X_{t}(T)$ satisfies

$$
d X_{t}(T)=\left(\eta^{2} / 2\right) X_{t}(T) d t+\eta d W_{t}
$$

Clearly, $S_{t}(T), t \geqslant 0$ is a martingale with initial value $S_{0}(T)$. 
The initial survival probability function $S_{0}(t)$ is assumed to be provided (in credit derivative applications, it is obtained by bootstrapping market quotes of financial instruments, like defaultable bonds or credit default swaps). It is decreasing and satisfies for all $t>0,0<S_{0}(t)<1$, which means the the associated hazard rate is strictly positive and finite. This leads to

$$
\begin{aligned}
S_{t}(T) & =\Phi\left(m(t, T)+\eta Z_{t}\right) \\
Z_{t} & :=\int_{0}^{t} e^{\left(\eta^{2} / 2\right)(t-s)} d W_{s} \\
m(t, T) & :=X_{0}(T) \mathrm{e}^{\left(\eta^{2} / 2\right) t}
\end{aligned}
$$

The Azéma supermartingale $S_{t}:=S_{t}(t), t \geqslant 0$ is often modeled either by using a simple Gaussian process Cesari et al. (2009) or by adopting the Cox process Brigo and Alfonsi (2005). However, the first approach clearly violates the $[0,1]$ condition, and the second corresponds to the specific case where $S$ is a decreasing predictable process. However, the general Doob-Meyer decomposition (in its additive form) reveals that in all generality, we have Bielecki et al. (2011), Profeta et al. (2010)

$$
d S_{t}=d D_{t}+d M_{t}
$$

where $D$ is a decreasing $\mathbb{F}$-predictable process and $M$ a martingale. The Cox setup is just one particular case. Moreover, it is hard to find a positive stochastic intensity model which allows for analytical calibration to market quotes whilst preventing negative path (in particular, square-root diffusion processes need to be shifted for calibration purposes, so that the resulting intensity process may not be positive anymore). This gives room to alternative modeling setups, and the $\Phi$-martingale is one of them Vrins (2014). The dynamics of the associated Azéma's martingale is proven to be

$$
\begin{aligned}
d S_{t} & =\zeta_{t} d S_{0}(t)+\eta \phi\left(\Phi^{-1}\left(S_{t}\right)\right) d W_{t} \\
\zeta_{t} & :=\frac{\phi\left(\Phi^{-1}\left(S_{t}\right)\right) \mathrm{e}^{\eta^{2} / 2 t}}{\phi\left(\Phi^{-1}\left(S_{0}(t)\right)\right)}
\end{aligned}
$$

\subsection{Unconditional survival probability and Azéma supermartingale}

The survival probability up to time $T \geqslant t$ given no default prior to time $t$ is obtained from Bayes' rule:

$$
Q_{t}(T):=\mathbb{E}\left[\mathbb{I}_{\{\tau>T\}} \mid \mathcal{F}_{t},\{\tau>t\}\right]=\frac{\mathbb{E}\left[\mathbb{I}_{\{\tau>(T \vee t)\}} \mid \mathcal{F}_{t}\right]}{\mathbb{E}\left[\mathbb{I}_{\{\tau>t\}} \mid \mathcal{F}_{t}\right]}=\frac{S_{t}(T)}{S_{t}(t)}
$$

which belongs to $[0,1]$ almost surely and is decreasing with respect to $T$ for all $T \geqslant t$. 


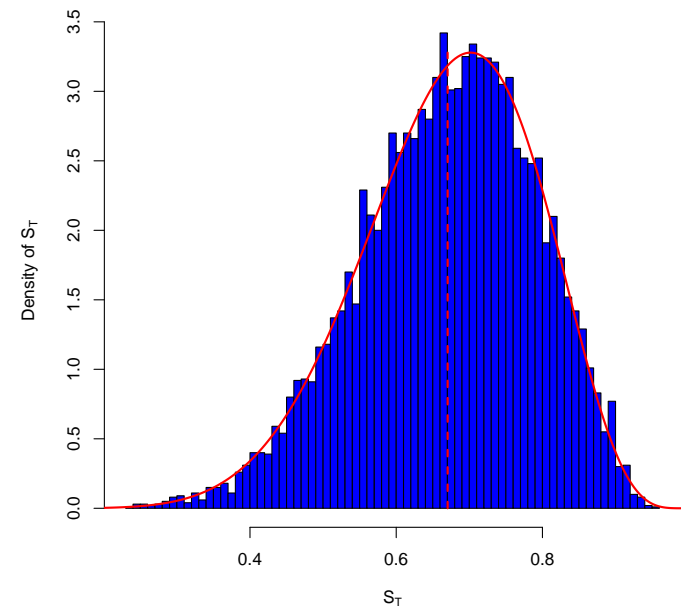

(a) Density of $S_{T}$. Histogram (10k paths, Euler discretization) and theoretical density obtained by differentiating $F_{Y_{t}}$ in (5.5) with $F=\Phi$ and $X_{t} \sim$ $\mathcal{N}\left(m(t, T)+\eta Z_{t}\right)($ red $)$

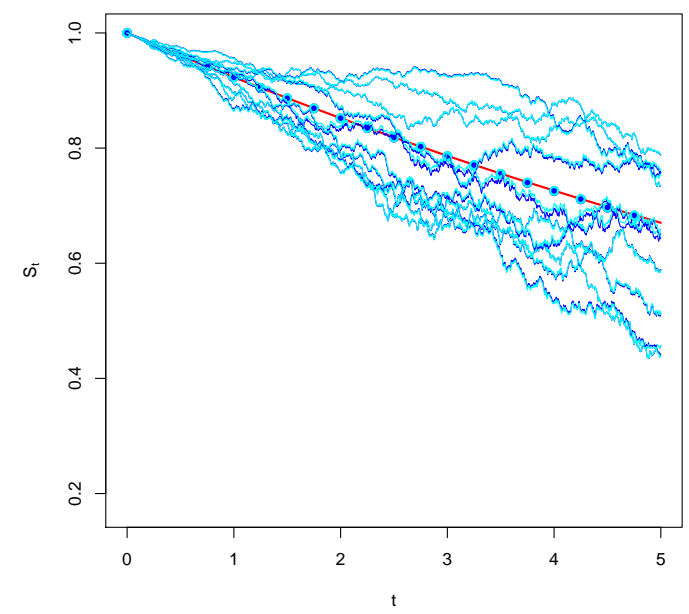

(b) Sample paths of the Azéma supermartingale $S_{t}$ using the analytical solution $\Phi\left(X_{t}\right)$ (cyan) and Euler discretization of $S_{t} \operatorname{SDE}(7.4)$ (dark blue)

Figure 3: Distribution of $S_{T}$ and sample paths of $S_{t}$. Parameters: $\eta=0.15, S_{0}(t)=\mathrm{e}^{-h t}, h=8 \%, T=$ 5.

We illustrate in Fig. 4 the distribution of $Q_{t}(T)$ for the 16 nodes $z_{i}$ associated to the 16-points Gauss-Hermite quadrature associated to the standard Normal factor $Z_{t}^{\star}=\eta Z_{t} / \sqrt{v(t)}$ where $Z_{t}$ is given by eq. (7.3) and $v(t)=\mathrm{e}^{\eta^{2} t}-1$ is the variance of $\eta Z_{t}$. If $Q(t, T ; z)$ stands for the value $Q_{t}(T)$ conditional upon $Z_{t}^{\star}=z$ and $\left(\omega_{i}, z_{i}\right), i \in\{1,2, \ldots, n\}$ are the weights and nodes of the $n$-points Gauss-Hermite quadrature, then

$$
\begin{aligned}
\mathbb{Q}_{0}\{\tau>T \mid \tau>t\} & =\mathbb{E}\left[Q_{t}(T)\right] \\
& =\mathbb{E}\left[Q\left(t, T, Z_{t}^{\star}\right)\right] \\
& \approx \sum_{i=1}^{n} \omega_{i} Q\left(t, T ; z_{i}\right) \\
Q(t, T ; z) & :=\frac{\Phi(m(t, T)+\sqrt{v(t)} z)}{\Phi(m(t, t)+\sqrt{v(t)} z)} \stackrel{(\eta \rightarrow 0)}{\rightarrow} \frac{S_{0}(T)}{S_{0}(t)}
\end{aligned}
$$

We consider the $t=0$ survival probability curve $S_{0}(t)=\mathrm{e}^{-\int_{0}^{t} h(s) d s}$ where the piece-wise constant hazard rate function $h(t)$ is given by the step function $\{t, \gamma(t)\}=\{(1,5 \%),(3,6 \%),(5,8 \%),(7,8.5 \%),(10,6.5 \%)\}$. One can see that the implied $Q\left(t, T ; z_{i}\right)$ curves can be quite different depending on the value of the driven factor, provided that the diffusion parameter $\eta$ of the Gaussian process underlying the $\Phi$-martingale is large enough. The boundary conditions for the cumulative distribution function $F_{Q_{t}(T)}(x)$ of $Q_{t}(T)$ are 


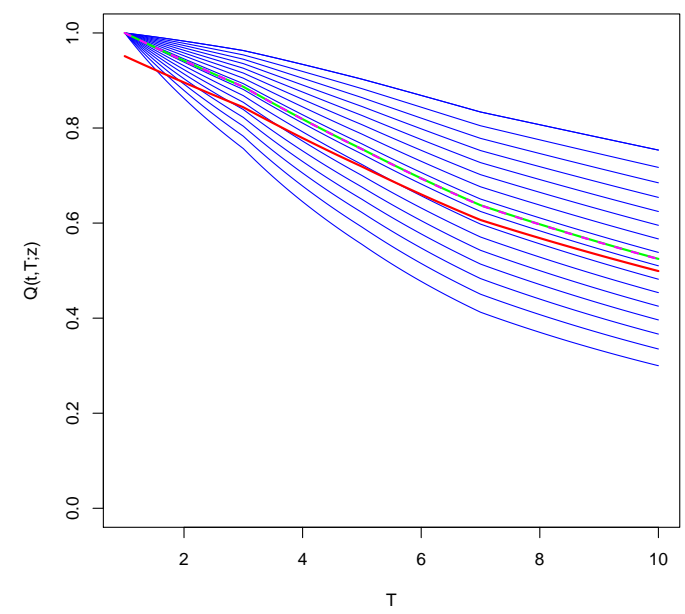

(a) $\eta=0.1$

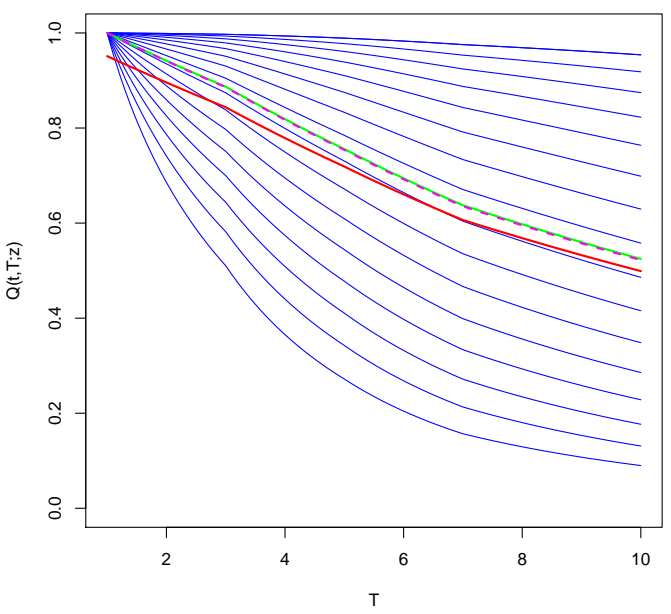

(b) $\eta=0.25$

Figure 4: $S_{0}(T)$ (red), $S_{0}(T) / S_{0}(t)$ (green), $Q\left(t, T ; z_{i}\right)$ (blue) and $\sum_{i=1}^{16} \omega_{i} Q\left(t, T ; z_{i}\right)$ (magenta).

$F_{Q_{t}(T)}(0)=0$ and $F_{Q_{t}(T)}(1)=1$.

For $x \in(0,1)$, it is proven to be (see Appendix 9.3)

$$
F_{Q_{t}(T)}(x)=\Phi\left(z^{\star}\right)
$$

where $z^{\star}=z^{\star}(t, T, x, \eta)$ is the (unique) root of the function

$$
G(z):=x \Phi(m(t, t)+\sqrt{v(t)} z)-\Phi(m(t, T)+\sqrt{v(t)} z)
$$

\subsection{Bivariate survival probability in the Gaussian copula setup}

Let us define the time- $t$ joint survival probability that $\tau_{1}>T_{1}$ and $\tau_{2}>T_{2}$ as

$$
G_{t}(\vec{T})=\mathbb{Q}\left\{\tau_{1}>T_{1}, \tau_{2}>T_{2} \mid \mathcal{F}_{t}\right\}
$$

We adopt a copula framework, where $G_{t}\left(T_{1}, T_{2}\right)$ depends on $t$ through the marginal (stochastic and correlated) distributions $S_{t, T_{1}}^{1}, S_{t, T_{2}}^{2}$ and time-dependent set of parameters $\Theta_{t}$ which is assumed to have finite variation ${ }^{7}$. In particular, the copula if fixed, but its parameter (e.g. correlation) can be timedependent:

$$
G_{t}(\vec{T})=C\left(S_{t}^{1}\left(T_{1}\right), S_{t}^{2}\left(T_{2}\right), \Theta_{t}\right)
$$

Observe that $G_{t}$ meets all the properties of multivariate cumulative distribution functions; this is guaranteed by the fact that we map valid margins through a copula $C(u, v, \Theta)$. However, it is clear

\footnotetext{
${ }^{7}$ Note the difference between $S_{i}(t)=S_{0}^{i}(t)=\mathbb{Q}\left\{\tau_{i}>t \mid \mathcal{F}_{0}\right\}$ and $S_{t}^{i}=S_{t}^{i}(t)=\mathbb{Q}\left\{\tau_{i}>t \mid \mathcal{F}_{t}\right\}$
} 
from eq. (7.6) that $G$ is a martingale, hence the SDE of $G$ must have no $d t$ term. This imposes some restrictions on the dynamics of the (meta) parameter $\Theta_{t}$.

Itô's lemma yields

$$
\begin{aligned}
d G_{t}(\vec{T})= & \frac{\partial C}{\partial u} d S_{t}^{1}\left(T_{1}\right)+\frac{\partial C}{\partial v} d S_{t}^{2}\left(T_{2}\right) \\
& +\frac{1}{2}\left(\frac{\partial^{2} C}{\partial u^{2}} d\left\langle S^{1} \cdot\left(T_{1}\right), S_{\cdot}^{1}\left(T_{1}\right)\right\rangle_{t}+\frac{\partial^{2} C}{\partial v^{2}} d\left\langle S_{\cdot}^{2}\left(T_{2}\right), S_{\cdot}^{2}\left(T_{2}\right)\right\rangle_{t}\right) \\
& +\frac{\partial^{2} C}{\partial u \partial v} d\left\langle S^{1}\left(T_{1}\right), S_{\cdot}^{2}\left(T_{2}\right)\right\rangle_{t}+\frac{\partial C}{\partial \Theta} d \Theta_{t}
\end{aligned}
$$

We now consider the bivariate Gaussian case, where correlated $\Phi$-martingales are plugged in a Gaussian copula:

$$
C(u, v, \Theta)=\Phi_{2}\left(\Phi^{-1}(u), \Phi^{-1}(v) ; r\right)
$$

with

$$
\begin{aligned}
S_{t}^{i}\left(T_{i}\right) & =\Phi\left(X_{t}^{i}\left(T_{i}\right)\right) \\
d X_{t}^{i}\left(T_{i}\right) & =\mu_{i} X_{t}^{i}\left(T_{i}\right) d t+\eta_{i} d W_{t}^{i} \\
d\left\langle W^{1}, W_{.}^{2}\right\rangle_{t} & =\rho d t
\end{aligned}
$$

Recall that $S_{t}^{i}\left(T_{i}\right)$ both need to be martingales, so that $\mu_{i}=\eta_{i}^{2} / 2$. Allowing the Gaussian copula (correlation) parameter to be a deterministic function of time $r(t)$,

$$
G_{t}(\vec{T})=\Phi_{2}\left(\Phi^{-1}\left(S_{t}^{1}\left(T_{1}\right)\right), \Phi^{-1}\left(S_{t}^{2}\left(T_{2}\right)\right) ; r(t)\right)=\Phi_{2}\left(X_{t}^{1}\left(T_{1}\right), X_{t}^{2}\left(T_{2}\right) ; r(t)\right)
$$

In this specific case, we get

$$
\begin{aligned}
d G_{t}(\vec{T})= & \frac{\partial \Phi_{2}}{\partial x} d X_{t}^{1}\left(T_{1}\right)+\frac{\partial \Phi_{2}}{\partial y} d X_{t}^{2}\left(T_{2}\right) \\
& +\frac{1}{2}\left(\frac{\partial^{2} \Phi_{2}}{\partial x^{2}} d\left\langle X_{\cdot}^{1}\left(T_{1}\right), X_{.}^{1}\left(T_{1}\right)\right\rangle_{t}+\frac{\partial^{2} \Phi_{2}}{\partial y^{2}} d\left\langle X_{\cdot}^{2}\left(T_{2}\right), X_{\cdot}^{2}\left(T_{2}\right)\right\rangle_{t}\right) \\
& +\frac{\partial^{2} C}{\partial x \partial y} d\left\langle X_{.}^{1}\left(T_{1}\right), X_{.}^{2}\left(T_{2}\right)\right\rangle_{t}+\frac{\partial \Phi_{2}}{\partial r} d r(t)
\end{aligned}
$$


The following derivatives are useful:

$$
\begin{aligned}
\frac{d \phi(x)}{d x} & =-x \phi(x) \\
\frac{\partial \Phi_{2}}{\partial r} & =\frac{\partial^{2} \Phi_{2}}{\partial x \partial y} \\
& =\frac{1}{\sqrt{1-r^{2}}} \phi(x) \phi\left(\frac{y-r x}{\sqrt{1-r^{2}}}\right) \\
& =g(x, y, r) \\
& =g(y, x, r) \\
\frac{\partial \Phi_{2}}{\partial x} & =\phi(x) \Phi\left(\frac{y-r x}{\sqrt{1-r^{2}}}\right) \\
& =h(x, y, r) \\
\frac{\partial^{2} \Phi_{2}}{\partial x^{2}} & =-x h(x, y, r)-r g(x, y, r)
\end{aligned}
$$

Noting that $d\left\langle X_{.}^{i}\left(T_{i}\right), X^{j}\left(T_{j}\right)\right\rangle_{t}=\rho_{i j} \eta_{i} \eta_{j} d t$ with $\rho_{i i}=1$ and $\rho_{i j}=\rho$, and $r^{\prime}(t)$ the derivative of $r(t)$, the dynamics of $G_{t}(\vec{T})$ become

$$
\begin{aligned}
d G_{t}(\vec{T})= & \eta_{1} h\left(X_{t}^{1}\left(T_{1}\right), X_{t}^{2}\left(T_{2}\right), r(t)\right) d W_{t}^{1}+\eta_{2} h\left(X_{t}^{2}\left(T_{2}\right), X_{t}^{1}\left(T_{1}\right), r(t)\right) d W_{t}^{2} \\
& +\left(r^{\prime}(t)+\rho \eta_{1} \eta_{2}-r(t) \frac{\eta_{1}^{2}+\eta_{2}^{2}}{2}\right) g\left(X_{t}^{1}\left(T_{1}\right), X_{t}^{2}\left(T_{2}\right), r(t)\right) d t
\end{aligned}
$$

Martingality is guaranteed provided that the $d t$ term is zero, that is

$$
r(t)=\frac{2 \rho \eta_{1} \eta_{2}}{\eta_{1}^{2}+\eta_{2}^{2}}+k \mathrm{e}^{\frac{\eta_{1}^{2}+\eta_{2}^{2}}{2} t}
$$

Because the first term is a constant but the second grows without bound, the $r(t) \in[-1,1]$ condition imposes $k=0$ if $\eta_{1}$ and $\eta_{2}$ are not both null. The only valid case is thus to set the Gaussian copula correlation parameter $r$ to the specific value $\frac{2 \rho \eta_{1} \eta_{2}}{\eta_{1}^{2}+\eta_{2}^{2}}$, depending on the variance and correlation of the latent processes underlying the marginal Azéma supermartingales.

Finally, the bivariate Azéma supermartingale has dynamics

$$
\begin{aligned}
d G_{t}(\vec{t}) & =\left.d G_{t}(\vec{T})\right|_{\vec{T}=(t, t)}+\xi_{t}^{1} d S_{0}^{1}(t)+\xi_{t}^{2} d S_{0}^{2}(t) \\
\xi_{t}^{1} & =\frac{\mathrm{e}^{\mu_{1} t} h\left(\Phi^{-1}\left(S_{t}^{1}\right), \Phi^{-1}\left(S_{t}^{2}\right), r(t)\right)}{\phi\left(\Phi^{-1}\left(S_{0}^{1}(t)\right)\right)} \\
\xi_{t}^{2} & =\frac{\mathrm{e}^{\mu_{2} t} h\left(\Phi^{-1}\left(S_{t}^{2}\right), \Phi^{-1}\left(S_{t}^{1}\right), r(t)\right)}{\phi\left(\Phi^{-1}\left(S_{0}^{2}(t)\right)\right)}
\end{aligned}
$$

where, $\xi_{t}^{i}$ are the multivariate equivalent to $\zeta_{t}$ and ensure that

$$
\mathbb{E}\left[G_{t}(t, t)\right]=G_{0}(t, t)=\Phi_{2}\left(\Phi^{-1}\left(S_{0}^{1}(t)\right), \Phi^{-1}\left(S_{0}^{2}(t)\right), r(0)\right)
$$


We conclude this section with Fig. 5 which illustrates the joint Azéma supermartingale processes $G_{t}(t, t)=$ $\mathbb{E}\left[\tau_{1}>t, \tau_{2}>t \mid \mathcal{F}_{t}\right]$ and the joint survival probability martingale $G_{t}(T, T)=\mathbb{E}\left[\left(\tau_{1} \wedge \tau_{2}\right)>T \mid \mathcal{F}_{t}\right]$ for different correlation levels (using the same pairs of paths of Brownian motions).

\section{Conclusion and future work}

In a first part of the paper, the conditions for a local martingale to be a genuine martingale have been reviewed and specialized to bounded processes. We have introduced the concept of conic processes as stochastic processes which range is finite and non-decreasing with respect to time. We have shown that martingales being locally bounded are in fact conic martingales. It has been explained how a martingale evolving between two constant bounds with given separable diffusion coefficient $\sigma(t, x)=g(t) h(x)$ can be obtained by mapping a stochastic process $X$ which diffusion coefficient is $g(t)$ through the function $F$ solving a first-order autonomous non-linear ODE featuring $h(x)$. This results is interesting for simulation purposes as the paths of $F(X)$ will then stay within the correct range.

The case of martingales evolving in the standard interval $[0,1]$ received specific attention. Several examples have been provided for which existence and uniqueness results have been established. The mapping $F$ consisting of the standard Normal cumulative distribution $\Phi$ proves to be interesting. It allows to turn any Itô integral into a martingale bounded in $[0,1]$. Moreover, the $\Phi$-martingale built by mapping a Vasicek process through $\Phi$ proves to be a tractable $[0,1]$-martingale that does not attain the bounds in finite time. Its statistics have been computed analytically, and its distribution is proven to converge to a Bernoulli with parameter given by the initial value of the process. It has been shown that it is the only $[0,1]$-martingale that can be obtained by mapping a specific class of Gaussian processes (called Gaussian diffusions) in a time-homogeneous way. The $\Phi$-martingale completes the class of the possible martingales that can be obtained by such means. The other processes are the constant, the rescaled Brownian motion and the driftless Geometric Brownian motion. Interestingly, each of these martingales correspond to a specific range.

Martingales in $[0,1]$ have been applied to the construction of Azéma supermartingales out of the Cox setup. They obviously meet the range constraint and benefits from automatic calibration. To our knowledge this is the first analytically tractable approach satisfying this requirement for all valid initial default probability curves. Similarly, one can built a set of conditional survival probability curves evolving in time with respect to a risk factor modeled as a Brownian motion. This was extended to the modeling of mutlivariate stochastic survival probabilities. 

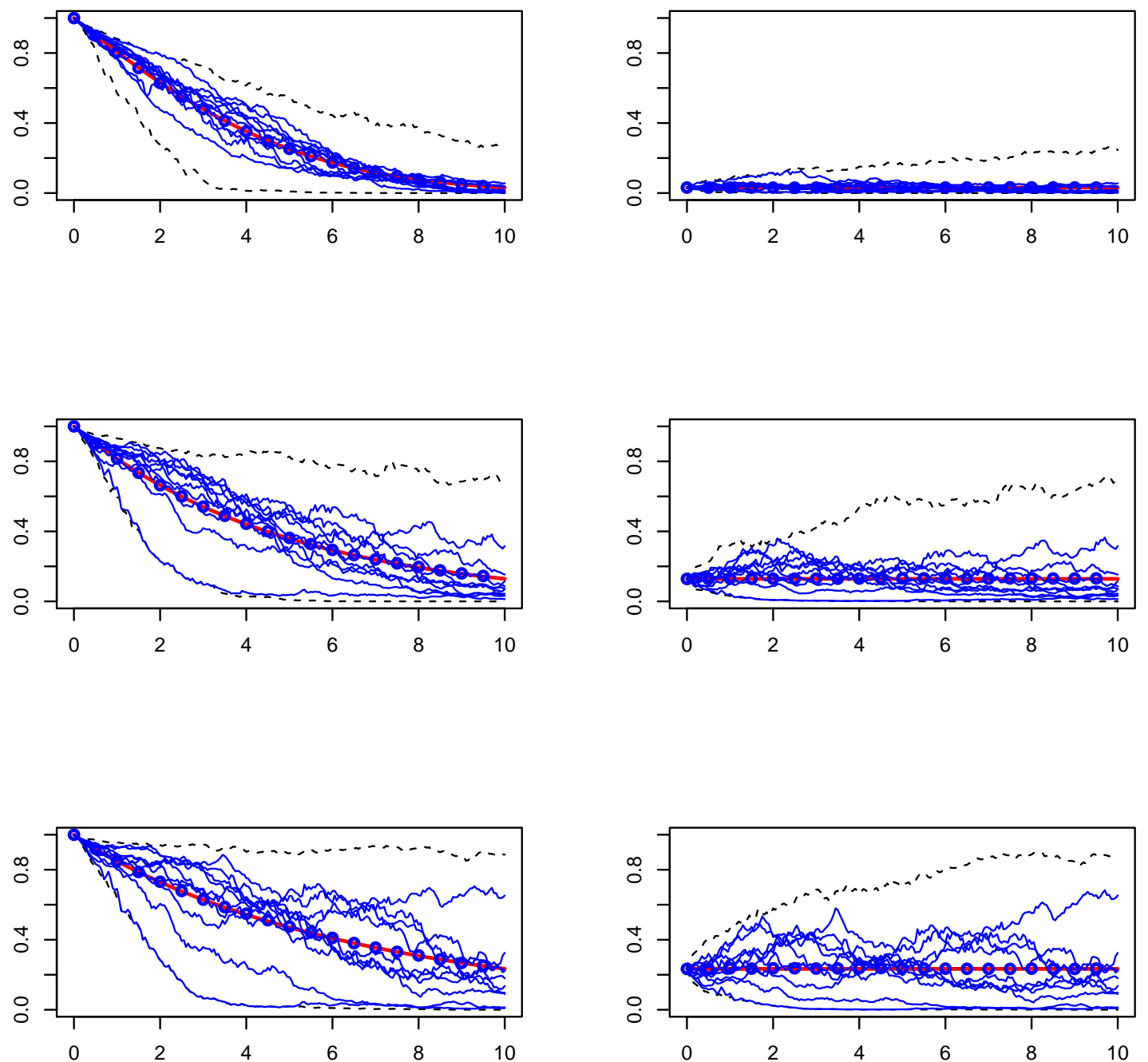

Figure 5: Bivariate Azéma supermartingale processes $G_{t}(t, t)=\mathbb{E}\left[\tau_{1}>t, \tau_{2}>t \mid \mathcal{F}_{t}\right]$ (left) and bivariate survival probability martingale $G_{t}(T, T)=\mathbb{E}\left[\left(\tau_{1} \wedge \tau_{2}\right)>T \mid \mathcal{F}_{t}\right]$ (right) with Brownian correlations $\rho=\{-80 \%, 0,+80 \%\}$ (top down). Ten sample paths are shown. There is no simulation error as the exact solution is known (up to numerical errors in the evaluation of the bivariate cumulative Normal distribution). We used constant hazard rates for $S_{0}^{i}(t)\left(h_{1}=8 \%\right.$ and $\left.h_{2}=12.5 \%\right)$, constant volatilities $\left(\eta^{1}=15 \%\right.$ and $\left.\eta^{2}=25 \%\right)$ time step of 0.05 . Blue dots on right panels show sample average based on 1,000 paths. Black dashed lines show the min and max envelopes based on the sample set. 
This work suggests several routes for future research. For instance, all the conic martingales derived in this paper have constant cones. Naive extension of the above construction schemes lead to SDEs that do not meet the usual existence criteria. It is not clear yet whether bounded martingales with timedependent cones can be found explicitly. Another route for future research deals with non-continuous bounded martingales. Finally, we believe this work opens the door for alternative approaches for the risk management of products depending on default probability curves, like for example the modeling of exposure profiles of credit-linked financial instruments.

\section{Appendix}

\subsection{Derivation of the law of $X_{t}$ in the Vehulst martingale $Y_{t}=\exp \left\{-\lambda X_{t}\right\}$}

Let us note the geometric Brownian motion $\Theta_{t}=g\left(t, W_{t}\right)$ where $g(t, x):=X_{0} \xi(t, x), \xi(t, x):=\exp \left\{\nu x-\frac{\nu^{2} t}{2}\right\}$ and let $\hat{\Theta}_{t}:=\int_{0}^{t} \Theta_{s} d s$. We seek for the density of $X_{t}=\Theta_{t} /\left(1-\mu \hat{\Theta}_{t}\right)$. To that end, we are interested in the joint density of $\left(\Theta_{t}, \hat{\Theta}_{t}\right)$. From the conditional density $p_{\hat{\Theta}_{t} \mid W_{t}}(y, x)$ of $\hat{\Theta}_{t}$ conditional upon the terminal value of the Brownian motion $W_{t}=x$, one gets

$$
f_{X_{t}}(z)=\int_{-\infty}^{\infty} p_{\hat{\Theta}_{t} \mid W_{t}}\left(\frac{z-g(t, x)}{\mu z} \mid x\right) \frac{\phi(x / \sqrt{t})}{\sqrt{t}} d x
$$

This expression features the density of the integral $\hat{\Theta}_{t}$ of a geometric Brownian motion conditional upon the terminal value of the Brownian motion $W_{t}$. This expression is quite important in finance, and appears in Asian options. Therefore, it received some attention and Marc Yor derived the corresponding expression by using relationships with Bessel processes Yor (1992).

Following Yor's notations, define

$$
A_{t}(\nu)=\int_{0}^{t} \exp \left\{2\left(W_{s}+\nu s\right)\right\} d s
$$

Then, observed that from the scaling property of Brownian motion,

$$
\int_{0}^{t} \exp \left\{a W_{s}+b s\right\} d s \sim \frac{4}{a^{2}} A_{a^{2} t / 4}\left(2 b / a^{2}\right)
$$

Setting $a=\eta, b=-\eta^{2} / 2, \nu=2 b / a^{2}=-1$ and $t^{\prime}=\eta^{2} t / 4$, we obtain

$$
\left(\hat{\Theta}_{t}, W_{t}\right) \sim\left(\frac{4 X_{0}}{\eta^{2}} A_{t^{\prime}}(-1), \frac{2}{\eta} W_{t^{\prime}}\right)
$$

On the other hand, if $\mathbf{Y}=\mathbf{A X}$ where $\mathbf{A}$ is an invertible matrix and $\mathbf{X}, \mathbf{Y}$ random (column) vectors, 
then the density of $\mathbf{Y}$ is given by $f_{\mathbf{Y}}(\mathbf{y})=\frac{1}{|\operatorname{det} \mathbf{A}|} f_{\mathbf{X}}\left(\mathbf{A}^{-1} \mathbf{y}\right)$. With $\mathbf{A}=\operatorname{Diag}\left(\frac{4 X_{0}}{\eta^{2}}, \frac{2}{\eta}\right)$,

$$
\begin{aligned}
f_{X_{t}}(z) & =\frac{\eta^{3}}{8 X_{0} \sqrt{t^{\prime}}} \int_{-\infty}^{\infty} p_{A_{t^{\prime}}(-1) \mid W_{t^{\prime}}}\left(\frac{\eta^{2}}{4 X_{0}}\left(\frac{z-g\left(t, \frac{\eta x}{2}\right)}{\mu z}\right) \mid \frac{\eta x}{2}\right) \phi\left(\frac{\eta x}{2 \sqrt{t^{\prime}}}\right) d x \\
& =\frac{\eta^{2}}{4 X_{0} \sqrt{t^{\prime}}} \int_{-\infty}^{\infty} p_{A_{t^{\prime}}(-1) \mid W_{t^{\prime}}}\left(\frac{\eta^{2}}{4 X_{0}}\left(\frac{z-g(t, y)}{\mu z}\right) \mid y\right) \phi\left(y / \sqrt{t^{\prime}}\right) d y
\end{aligned}
$$

Yor argued that it is enough to study the conditional law of $A_{t}(0)$ since $p_{A_{t}(\nu) \mid W_{t}}\left(z \mid y+\nu_{t}\right)=p_{A_{t}(0) \mid W_{t}}(z \mid y)$. To see this, let us define the measure $\tilde{\mathbb{Q}}$ according to the Radon-Nikodym derivative process $\left.\frac{d \tilde{\mathbb{Q}}(\omega)}{d \mathbb{Q}(\omega)}\right|_{\mathcal{F}_{t}}=$ $\xi\left(t, W_{t}\right)$. From Girsanov's theorem, $\tilde{W}_{t}=W_{t}+\nu t$ is a $\tilde{\mathbb{Q}}$-Brownian motion, and the claim follows:

$$
\begin{aligned}
p_{A_{t}(\nu) \mid W_{t}}(z \mid y) & =\frac{\int_{\Omega} \mathbb{I}_{\left\{A_{t}(\nu)=z, W_{t}=y\right\}} d \mathbb{Q}(\omega)}{\int_{\Omega} \mathbb{I}_{\left\{W_{t}=y\right\}} d \mathbb{Q}(\omega)} \\
& =\frac{\int_{\Omega} \mathbb{I}_{\left\{\int_{0}^{t} \exp \left\{2 \tilde{W}_{s}\right\} d s=z, \tilde{W}_{t}=y+\nu t\right\}} d \mathbb{Q}(\omega)}{\int_{\Omega} \mathbb{I}_{\left\{\tilde{W}_{t}=y+\nu t\right\}} d \mathbb{Q}(\omega)} \\
& =\frac{\int_{\Omega} \mathbb{I}_{\left\{\int_{0}^{t} \exp \left\{2 \tilde{W}_{s}\right\} d s=z, \tilde{W}_{t}=y+\nu t\right\}} \xi(t, y) d \tilde{\mathbb{Q}}(\omega)}{\int_{\Omega} \mathbb{I}_{\left\{\tilde{W}_{t}=y+\nu t\right\}} \xi(t, y) d \tilde{\mathbb{Q}}(\omega)} \\
& =\frac{\xi(t, y) \int_{\Omega} \mathbb{I}_{\left\{A_{t}(0)=z, W_{t}=y+\nu t\right\}} d \mathbb{Q}(\omega)}{\xi(t, y) \int_{\Omega} \mathbb{I}_{\left\{W_{t}=y+\nu t\right\}} d \mathbb{Q}(\omega)} \\
& =p_{A_{t}(0) \mid W_{t}}(z \mid y+\nu t)
\end{aligned}
$$

Finally, the density $f_{X_{t}}$ is obtained from the law of $A_{t}(0)$ conditioned upon the terminal value of the Brownian motion $W_{t}, a_{t}(y, z)=p_{A_{t}(0) \mid W_{t}}(z \mid y)$, which is proven in Yor (1992) to be:

$$
\begin{aligned}
a_{t}(y, z) & =\frac{\sqrt{t}}{z \phi(y / \sqrt{t})} \exp \left(-\frac{1+e^{2 y}}{2 z}\right) \theta_{e^{y} / z}(t) \\
\theta_{r}(u) & =\frac{1}{\sqrt{2 u \pi^{3}}} \exp \left(\frac{\pi^{2}}{2 u}\right) \Psi_{r}(u) \\
\Psi_{r}(u) & =\int_{0}^{\infty} \exp \left(\frac{-y^{2}}{2 u}\right) \exp (-r \cosh y) \sinh (y) \sin \left(\frac{\pi y}{u}\right) d y
\end{aligned}
$$

and we obtain

$$
f_{X_{t}}(z)=\frac{\eta^{2}}{4 X_{0} \sqrt{t^{\prime}}} \int_{-\infty}^{\infty} a_{t^{\prime}}\left(y-t^{\prime}, \frac{\eta^{2}}{4 X_{0}}\left(\frac{z-g\left(t^{\prime}, y-t^{\prime}\right)}{\mu z}\right)\right) \phi\left(y / \sqrt{t^{\prime}}\right) d y
$$

\subsection{Collapsing property of bounded martingales}

It has been proven that when $X$ is a Gaussian diffusion with diffusion coefficient $\eta$ and $\operatorname{drift}\left(\eta^{2} / 2\right) x$ then $Y=\Phi(X)$ is a martingale bounded in $[0,1]$ which converges in distribution to a $\operatorname{Bernoulli}\left(Y_{0}\right)$. This proof was easy as the distribution of $Y_{t}$ is known analytically. However, it is likely that autonomous martingales of the form $F(X)$ where $X$ is a free process and the image of $F$ is a compact interval $[a, b]$ will share the same "collapsing" feature. Although we do not give a formal proof, we provide an intuitive development below. We further discuss which form of mappings $F$ could potentially not have this feature. 
Let $F$ (assumed to be strictly increasing and $\mathcal{C}^{2}$ ) and $f$ be unimodal (i.e. $f^{\prime}(x)$ is first positive, then vanishes at some point $x^{\star}$ and then remains negative) when $\eta\left(t, X_{t}\right)=\eta$. Recall the SDE followed by $X$ :

$$
d X_{t}=-\frac{\eta^{2}}{2} \frac{f^{\prime}\left(X_{t}\right)}{f\left(X_{t}\right)} d t+\eta d W(t)=\frac{\eta^{2}}{2} \psi\left(X_{t}\right) d t+\eta d W(t)
$$

Because $f^{\prime}(x)<0$ for all $x>x^{\star}$ and $f^{\prime}(x)>0$ for all $x>x^{\star}$, we can see from the above SDE that $X$ has a positive drift when being above $x^{\star}$ and a negative drift otherwise. As long as we choose $F$ such that $f$ is unimodal, then the process will tend to diverge, and same will hold true for $Y=F(X)$. In other words, conic martingales $Y$ obtained via an underlying process $Y=F(X)$ will be attracted towards one of the boundaries when the bounds $(a, b)$ are constant and $f=F^{\prime}$ is unimodal.

Although it could take quite some time before $Y$ collapses to $a$ or $b$, it is very likely to happen, unless $F$ is chosen to have some specific properties, and the above development allows us to understand which properties may break this attraction.

A first possibility of course is to use a vanishing time-dependent diffusion coefficient $\eta(t)$. If $\eta(t)$ collapses to zero, the process $X$ will be frozen, and so are the paths of the $Y_{t}$ process. However, we argue that this is not the only way to prevent all paths to converge to one of the bounds. Although we do not give formal proof, we claim that this can be achieved by choosing $F$ so that $F^{\prime}=f$ is bimodal (i.e. $f^{\prime}$ changes sign between the two modes) and $X_{0}$ belongs to the interval defined by the two modes. Consider for example $F(x)=1 / 2\left(\Phi\left(\frac{x-\left(X_{0}+\mu\right)}{s}\right)+\Phi\left(\frac{x-\left(X_{0}-\mu\right)}{s}\right)\right)$ for $s>0$ and $\mu$ large enough to ensure that the sign of $f^{\prime}$ changes between the two modes. This function maps $\mathbb{R}$ to the unit interval $[0,1]$. Then, the fact that $X_{t}$ falls in the "dip" $\left[X_{0}-\mu, X_{0}+\mu\right]$ will create a pulling effect such that $X_{t}$ will tend to stay within this interval. ${ }^{8}$ The effect of the bimodal nature of $f$ is to partly prevent all paths to collapse to one of the boundaries (the center of the distribution would not be empty anymore; the probability to be in arbitrarily small neighborhood of the bounds would be non-zero, but would not sum to 1 either). It remains to formally prove that $\mathbb{Q}\left\{X_{t} \in\left[X_{0}-\mu, X_{0}+\mu\right]\right\}>0$ as $t \rightarrow \infty$ for any $\mu>0$. This is cumbersome since we do not have an analytical expression for the distribution of $X_{t}$. However, Monte Carlo simulations or PDE solver seem to confirm that this is effectively the case: only part of the paths collapse to the boundary. The "sharpness of the dip" (which can be tuned by playing with $s$ ) determines the probability that $Y_{t}$ lies in $\left[F\left(X_{0}-\mu\right), F\left(X_{0}+\mu\right)\right]$ as $t \rightarrow \infty$. However, the process $X_{t}$ built according to the above procedure has a stable stationary point (zero drift, or equivalently $f^{\prime}(x)=0$ ) at $X_{0}$ (zero drift, and in the neighborhood, the drift tends to pull $X_{t}$ back to $X_{0}$ ), and unstable stationary points

\footnotetext{
${ }^{8}$ Observe that this pulling effect does not impact the martingale property of $Y$, since this is compensated by the function $F$, just like the fact that $X$ is a diverging process when $F=\Phi$ is used did not impact the martingality of $Y$ in the unimodal case.
} 
at $X_{0} \pm \mu$ (zero drift, but when $X_{t}$ moves around these points, the effect of the drift is to push $X_{t}$ away from those). It is then likely that the paths, instead of collapsing (asymptotically) to either 0 or 1 almost surely, now asymptotically collapse to $\{0,1\}$ with some probability $p$, but $X_{t}$ has a non-zero probability $1-p$ to be in the interval $\left[X_{0}-\mu, X_{0}+\mu\right]$ even in the limit $t \rightarrow \infty$. In particular, we expect to have $\lim _{t \rightarrow \infty} \mathbb{Q}\left\{Y_{t} \in\left(0, F\left(X_{0}-\mu\right)\right]\right\}=\lim _{t \rightarrow \infty} \mathbb{Q}\left\{\left[Y_{t} \in\left[F\left(X_{0}+\mu\right), 1\right)\right\}=0\right.$, while for any $\epsilon>0$, $\lim _{t \rightarrow \infty} \mathbb{Q}\left\{Y_{t} \in(1-\epsilon, 1]\right\}=\lim _{t \rightarrow \infty} \mathbb{Q}\left\{Y_{t} \in[0, \epsilon)\right\}>0$ and $\lim _{t \rightarrow \infty} \mathbb{Q}\left\{Y_{t} \in\left[F\left(X_{0}-\mu\right), F\left(X_{0}+\mu\right)\right]\right\}>0$.

\subsection{Distribution of $Q_{t}(T)$}

In this section we show that for $x \in(0,1), \eta^{2}<\infty$ and $S_{0}(T)<S_{0}(t)$, the cumulative distribution function $F_{Q_{t}(T)}(x)$ of $Q_{t}(T)$ is given by $\Phi\left(z^{\star}(t, T, x, \eta)\right)$.

First, we notice that $\eta Z_{t} \sim \sqrt{v(t)} Z$ where $Z$ a standard Normal variable, the distribution function of $Q_{t}(T)$ is given by

$$
\begin{aligned}
\mathbb{Q}\left\{Q_{t}(T) \leqslant x\right\} & =\mathbb{Q}\{Q(t, T ; Z) \leqslant x\} \\
& =\mathbb{Q}\{\Phi(m(t, T)+\sqrt{v(t)} Z) \leqslant x \Phi(m(t, t)+\sqrt{v(t)} Z)\} \\
& =\mathbb{Q}\{\Phi(y) \leqslant x \Phi(m+y)\} \\
y & :=m(t, T)+\sqrt{v(t)} Z \\
m & :=m(t, t)-m(t, T)
\end{aligned}
$$

From the above notations, we can write

$$
F_{Q_{t}(T)}(x)=\int_{z: G(z)>0} d \Phi(z)
$$

Moreover, by definition of $z^{\star}, G\left(z^{\star}\right)=0$. It remains to show that $\{z: G(z)>0\}=\left(-\infty, z^{\star}\right]$, in which case we have the claim

$$
\int_{z: G(z)>0} d \Phi(z)=\Phi\left(z^{\star}\right)
$$

Clearly, under our assumptions on $S_{0}(T), m>0$. We define

$$
\tilde{G}(y)=x \Phi(m+y)-\Phi(y)
$$

so that $\tilde{G}(y)=\tilde{G}(m(t, T)+\sqrt{v(t)} z)=G(z)$. Our purposes is to show that for $m>0$ and $x \in(0,1)$, $\{y: \tilde{G}(y)>0\}=\left(-\infty, y^{\star}\right]$ with $z^{\star}=\left(y^{\star}-m(t, T)\right) / \sqrt{v(t)}$, since the claim then results from a continuity argument.

First, we notice that $\tilde{g}(y)=d \tilde{G}(y) / d y$ has one single root, $y_{0}=\ln (x) / m-m / 2$, and $\tilde{g}(y)>0$ for $y<y_{0}$ whilst $\tilde{g}(y)<0$ for $y>y_{0}$. Because $\tilde{G}(y)=\int_{-\infty}^{y} \tilde{g}(u) d u$ (the integration constant is zero as 
$\lim _{y \downarrow-\infty} \tilde{G}(y)=0$ ), the smallest root of $\tilde{G}, y_{1}^{\star}$ is larger than $y_{0} ; y_{1}^{\star}>y_{0}$. However, for $y>y_{0}, \tilde{g}(y)<0$, meaning that on the right of its first root, $\tilde{G}(y)$ is strictly decreasing from 0 . Function $\tilde{G}(y)<0$ for $y>y_{1}^{\star}$, showing that if it exists, $y_{1}^{\star}$ is the unique root $y^{\star}$ of the continuous function $\tilde{G}$ or equivalently, that $G$ admits a unique root $z^{\star}=\left(y^{\star}-m(t, T)\right) / \sqrt{v(t)}$.

\section{References}

G. Cesari, J. Aquilina, N. Charpillon, Z. Filipovic, G. Lee, and I Manda. Modelling, pricing and hedging counterparty credit exposure - A Technical Guide. Springer, 2009.

F. Vrins. Conic martingales. In 8th World Congress of the Bachelier Finance Society, Brussels, June 2014.

F. Vrins and M. Jeanblanc. The $\phi$-martingale. In International Colloquium of Actuarial Association, Oslo, June 2015.

A.M Cox and D. Hobson. Local martingales, bubbles and option prices. Finance and Stochastics, 9(4): 477-492, 2005.

P. Protter. A mathematical theory of financial bubbles. In Paris-Princeton Lecture Notes in Mathematical Finance 2013, volume 2081 of Lecture Notes in Mathematics, pages 1-108. Springer, 2013.

I. Karatzas and S. Shreve. Brownian Motion and Stochastic Calculus. Springer, 2005.

D. Revuz and M. Yor. Continuous martingales and Brownian motion. Springer-Verlag, New-York, 1999.

S.E. Shreve. Stochastic Calculus for Finance vol. II - Continuous-time models. Springer, 2004.

A.K. Zvonkin. A transformation of the phase space of a process that removes the drift. Math. USSR Sbornik, 93(1):129-149, 1974.

P. E. Kloeden and E. Platen. Numerical Solution of Stochastic Differential Equation. Applications of Mathematics (stochastic modelling and applied probability)). Springer, 1999.

M. Jeanblanc, M. Yor, and M. Chesney. Martingale Methods for Financial Markets. Springer Verlag, Berlin, 2007.

P. Carr, A. Cherny, and M. Urusov. On the martingale property of time-homogeneous diffusions. Technical report, 2007. URL http://homepage.alice.de/murusov/papers/ccu-mart.pdf. 
F. Delbaen and H. Shirakawa. No arbitrage condition for positive diffusion price processes. Asia-Pacific Financial Markets, 9:159168, 2002.

C. Dellacherie and Meyer P.-A. Probabilités et Potentiel - Espaces Mesurables. Hermann, 1975.

D. Veneziano. Envelopes of vector random processes and their corssing rates. Ann. Prob., 7(1):62-74, 1979.

P. Protter. Stochastic Integration and Differential Equations. Springer, Berlin, Second edition, 2005.

B. Øksendal. Stochastic Differential Equations. An Introduction with Applications. Universitext. Springer, 2003.

M. Yor. On some exponential functionals of Brownian motion. Advances in Applied Probability, 24(3): $509-531,1992$.

D. Lando. Credit Risk Modeling: Theory and Applications. Pinceton Series in Finance. Pinceton University Press, 2004.

T. Bielecki, M. Jeanblanc, and M. Rutkowski. Credit risk modeling. Technical report, Osaka (Japan), 2011.

D. Brigo and A. Alfonsi. Credit default swaps calibration and option pricing with the SSRD stochastic intensity and interest rate model. Finance and Stochastics, 9:29-42, 2005.

C. Profeta, B. Roynette, and M. Yor. Option Prices as Probabilities: A New look at Generalized BlackScholes Formulae. Springer Finance, 2010. 